\title{
Cladistics
}

Cladistics 35 (2019) 351-384

$10.1111 /$ cla. 12363

\section{Phylogeny of the family Cladoniaceae (Lecanoromycetes, Ascomycota) based on sequences of multiple loci}

\author{
Soili Stenroos ${ }^{\mathrm{a},{ }^{*} \dagger}$, Raquel Pino-Bodas ${ }^{\mathrm{b},{ }^{*} \dagger, \dagger}$, Jaakko Hyvönen $^{\mathrm{a}}$, Helge Thorsten Lumbsch ${ }^{\mathrm{c}}$ \\ and Teuvo Ahti ${ }^{\mathrm{a}}$ \\ ${ }^{a}$ Finnish Museum of Natural History, Botany Unit, University of Helsinki, PO Box 47, FI-00014, Helsinki, Finland; ${ }^{b}$ Real Jardín Botánico, CSIC, \\ Plaza de Murillo 2, 28014, Madrid, Spain; 'Science \& Education, The Field Museum, 1400 S. Lake Shore Drive, Chicago, IL, 60605, USA
}

Accepted 15 October 2018

\begin{abstract}
Cladoniaceae is a family of lichenized fungi that belongs to the Lecanorales, Ascomycota. The family is distributed widely, although several genera are restricted to the Southern Hemisphere. The circumscriptions of the genera and species in the family have traditionally been based on thallus morphology, the type of vegetative propagules and the secondary metabolites. However, numerous species are highly variable phenotypically, making their delimitation problematic. In the present study a new phylogeny of Cladoniaceae is constructed using five loci (ITS rDNA, IGS rDNA, RPB2, RPB1,EF-1a) from a worldwide sample of 643 specimens representing 304 species. Cladoniaceae was resolved as a monophyletic group. The circumscription of the genera and the relationships among them are discussed. Pycnothelia, Carassea and Metus are closely related, forming a sister clade to the larger genus Cladonia. Cladia in its recent wide sense turned out to be paraphyletic, including species that have been recognized in Thysanothecium and Notocladonia. Cladonia was resolved as monophyletic, with C. wainioi as the earliest diverging lineage. Eleven major clades were resolved in Cladonia. No synapomorphies were found for most of them. We propose the new genera Pulchrocladia and Rexia, as segregates of Cladia, five new combinations, and the resurrection of the genus Heterodea.

(C) The Willi Hennig Society 2018.
\end{abstract}

\section{Introduction}

Cladoniaceae (Lecanorales, Ascomycota) is one of the largest families of lichen-forming fungi (Lücking et al., 2016), with almost 500 accepted species in 15 genera (Wijayawardene et al., 2018). Numerous phylogenetic studies have shown that Cladoniaceae belongs to the Lecanorales, and is closely related to Stereocaulaceae (Wedin et al., 2000; Stenroos et al., 2002b; Miadlikowska et al., 2006; Arup et al., 2007; Ekman et al., 2008; Miadlikowska et al., 2014). In a recent classification based on a temporal approach,

*Corresponding authors:

E-mail address: soili.stenroos@helsinki.fi; rpino@rjb.csic.es; r.pino@ kew.org

${ }^{\dagger}$ These authors contributed equally to this study.

Current address: Royal Botanical Garden, Kew, Richmond TW9 $3 \mathrm{AB}, \mathrm{UK}$.
Stereocaulaceae was included in an enlarged concept of Cladoniaceae (Kraichak et al., 2018). Cladoniaceae was formally introduced by Zenker in Goebel (1827-1829), but its delimitation has varied over time. The number of species assigned to the family has increased notably since the monographer of the family, E. A. Vainio (Wainio), published his three-volume opus (Vainio, 1887, 1897) with 134 species and subspecies. The family's taxonomic landmarks have shifted over time. The genus Cladia was accepted as distinct from Cladonia after Filson's (1981) monograph; Neophyllis was moved to Sphaerophoraceae (Wedin and Döring, 1999); and Heterodea, at first placed in Parmeliaceae (Reinke, 1895; Zahlbrucker, 1926) and later in its own monotypic family Heterodeaceae (Filson, 1978), was shown to belong to Cladoniaceae using molecular data (Wedin et al., 2000). Similarly, Pilophorus, placed for a long time in Stereocaulaceae (Reinke, 1895; Jahns, 
1970a, 1981; Henssen and Jahns, 1973; Tehler, 1996), was later included in Cladoniaceae (Stenroos and DePriest, 1998; Wedin et al., 2000). Cladonia connexa was separated into its own genus Carassea by Stenroos et al. (2002b). The status of the so-called reindeer lichens has varied from a separate genus Cladina, to a subgenus of Cladonia (Ruoss and Ahti, 1989; Stenroos et al., 1997; Ahti, 2000). Currently, Cladina species are included in Cladonia without a formal taxonomic rank (Stenroos et al., 2002a, 2015). Cetradoniaceae was merged with Cladoniaceae (Zhou et al., 2006) and, most recently, Heterodea and Ramalinora were included in Cladia (Lumbsch et al., 2010a; Parnmen et al., 2010).

Many taxa of the Cladoniaceae are conspicuous fruticose or foliose lichens, dominant in many habitats and on a diversity of substrates (soil, rocks, tree trunks, rotten wood), including the Arctic and Antarctic tundra, boreal and antiboreal forests, bogs, temperate forests, pioneer habitats (e.g. roadsides), tropical highlands, and even the sandy tropical lowlands of Amazonia. However, they are absent from very arid regions. Species in Cladoniaceae are commonly characterized by a dimorphic thallus, with a squamulose or crustose primary thallus that can be permanent or evanescent, and a secondary fruticose thallus termed a podetium or pseudopodetium according to their ontogenetic origin (Jahns, 1970a; Hammer, 1995). The podetia or pseudopodetia of different species range from a few millimetres high to conspicuous thalli $25-30 \mathrm{~cm}$ tall. Some genera, however, lack either a primary thallus or fruticose structures. Ascomata are biatorine apothecia, often dark brown, sometimes pale brown, red, ochraceous or black. Cladoniaceae asci represent a variant of the Porpidia type, with a contrasting tube-like amyloid reaction pattern of the ascus apex almost all producing eight simple, hyaline, ellipsoidal spores per ascus. Unlike other lichen groups, their hymenium has few features useful as taxonomic characters. Exceptions are found in Calathaspis, Pycnothelia and Pilophorus (Lamb et al., 1972; Jahns, 1981; Galloway and James, 1987), which can produce septate ascospores. However, not all hymenial details are well studied. For example, Gymnoderma has an entirely amyloid ascus tip structure (Peršoh et al., 2004), so delimiting characteristics remain to be discovered. Conversely, the ontogeny of the ascomata has been studied in detail in most genera of Cladoniaceae (Jahns, 1970a; Jahns and Beltman, 1973; Hammer, 1993; Jahns et al., 1995; Grube and Hawksworth, 2007), although the interpretation of some structures has been controversial (Hammer, 1993, 1995).

Most members of Cladoniaceae form a bipartite symbiotic association with the green algae Asterochloris (Tschermak-Woess, 1980; Piercey-Normore and DePriest, 2001; Peršoh et al., 2004; Moya et al., 2015), or more rarely Chlorella (Peršoh et al., 2004), both members of Trebouxiophyceae. The genus Trebouxia s.s., commonly found in other lichens, has not been recorded in Cladoniaceae (Peršoh et al., 2004; Moya et al., 2015). However, the photobionts have not been examined in all genera. Cyanobacteria (Nostoc and Stigonema) are present only in the genus Pilophorus (Jahns, 1981), and are located in separate structures called cephalodia.

Cladoniaceae is exceptionally rich in secondary metabolites (Culberson, 1969, 1970; Culberson et al., 1977; Huovinen and Ahti, 1982, 1986a,b, 1988; Huovinen et al., 1989a,b, 1990; Ahti, 2000), with more than 70 different substances reported. Most are depsides and depsidones (polyphenolics), but terpenoids and aliphatic acids also are common (Huovinen and Ahti, 1986a,b, 1988; Huovinen et al., 1990; Huneck and Yoshimura, 1996). The composition of secondary metabolites varies among species, and numerous species produce multiple chemotypes.

Traditionally, taxonomy in Cladoniaceae at the species level has been based on the morphology of podetia or pseudopodetia: overall shape, cup shape, tip shape, branching type, presence/absence of squamules, soredia or cortex, or anatomical characters (Ahti, 2000). Certain secondary metabolites were also considered useful in species identification (Dahl, 1952; Huovinen and Ahti, 1982; Culberson, 1986). Extensive taxonomic background data for the classification and characteristics of the family or its genera can be found in Thomson (1968), Filson (1981), Jahns (1981), Ahti (2000), Burgaz and Ahti (2009), Galloway (2007), and Ahti and Stenroos (2013), among others.

Cladonia is by far the largest and most diverse genus of Cladoniaceae, currently with about 475 accepted species. New species continue to be described, even though the genus is among the most thoroughly studied large lichen groups globally. Unlike the other genera, which have more restricted distributions, Cladonia is practically cosmopolitan (Ahti, 2000). Cladonia has been divided over time into numerous formally named subdivisions, such as subgenera, sections and subsections, which have been applied in various ways at different times (Vainio, 1897; Sandstede, 1931; Mattick, 1938, 1940; Dahl, 1952; Thomson, 1968). Ahti (2000) classified species into seven sections, Ascyphiferae, Cocciferae, Cladonia, Helopodium, Perviae, Strepsiles and Unciales, while the sections Cladina, Impexae and Tenues were placed in Cladina. These sections were circumscribed using a combination of morphological and/ or chemical characteristics, such as red apothecia containing rhodocladonic acid, funnel-like branch axils, or various branching podetial patterns. Although this classification treated primarily the Latin American species, Ahti (2000) assumed that most Cladonia species could be placed within these sections. However, Stenroos et al. (2002a) demonstrated using two DNA markers that most sections were not monophyletic. Furthermore, only a few clades could be delimited 
accurately based on phenotypic characteristics. Anticipating more robust data in the future, Stenroos et al. (2002a) applied informal names for the major clades (three divisions, four supergroups and six groups).

Cladia is the second largest genus of the family with 23 species according to recent revisions (Parnmen et al., 2012, 2013). It is distributed mainly in the Southern Hemisphere but extends into South-East Asia and the Neotropics towards the Northern Hemisphere. It has been circumscribed by variably branched, perforated pseudopodetia (Filson, 1981). The phylogenetic studies of Parnmen et al. (2010) and Lumbsch et al. (2010a) showed three well-supported clades. Parnmen et al. (2012) tested species delimitation of the phenotypically variable Cladia aggregata complex and found several distinct lineages. Similarly to Cladonia, morphological and chemical characteristics often did not delimit the lineages, with many apparently cryptic species (i.e. not recognizable without DNA analyses). However, several new species could be distinguished with a combination of phenotypic characters and patterns of geographical distribution (Parnmen et al., 2013). The group needs further, thorough treatment using material from across its entire geographical range.

While Cladoniaceae is among the best studied lichen families, and amongst the first with a molecular phylogeny, the taxonomy and phylogenetic placement of many genera and species (DePriest, 1993, 1994; Stenroos and DePriest, 1998; DePriest et al., 2000; Wedin et al., 2000) remain poorly known. The most extensive phylogeny by Stenroos et al. (2002a) included 153 species, representing $32.2 \%$ of all accepted species in Cladoniaceae at the time. They used two markers, ITS rDNA and $\beta$-tubulin, along with chemical and morphological characters to estimate their phylogeny. A further phylogenetic analysis with more extensive taxon sampling and additional markers is necessary to study the relationships within this family.

Our principal aim was to examine the phylogenetic relationships of Cladoniaceae, and in particular to explore the generic delimitation and the relationships among the species of the largest genus Cladonia. Compared to the earlier analyses of Cladonia, we increased taxon sampling to be more globally representative, and we added a number of markers that are frequently used in the systematics of Ascomycota (Reeb et al., 2004; Hofstetter et al., 2007; Schoch et al., 2009; Miadlikowska et al., 2014).

\section{Material and methods}

\section{Taxon sampling}

For the present study, we used sequence data and available extractions remaining from the study by
Stenroos et al. (2002a). Additional samples were collected from areas such as New Zealand, Scotland and the Azores. The sampling was completed, with material taken from the Helsinki herbarium $(\mathrm{H})$, other herbaria (CANB, F, KW, MACB), or provided by colleagues. In total, we included 643 specimens from 304 species of Cladoniaceae, representing ten of the 15 known genera (Table S1). The sampling included 289 species (ca.61\%) of Cladonia representing all the Cladonia sections (sensu Ahti, 2000). Unfortunately, we were not able to obtain fresh material of the small genera Calathaspis, Heteromyces, Sphaerophoropsis and Squamella (Lumbsch and Huhndorf, 2010), and for numerous species of Cladonia, mainly from tropical areas. The missing genera are rare, most of them only known from few localities, and most are monotypic. Twelve ITS rDNA sequences of rare Cladonia species were downloaded from GenBank. As outgroups, three species of Lepraria (L. membranacea, L. incana and L. jackii) and six species of Stereocaulon (S. alpinum, $S$. nanodes, S. paschale, S. tomentosum, S. sasakii and $S$. spathuliferum) were selected, based on the results of Stenroos et al. (2002b), Myllys et al. (2005) and Miadlikowska et al. (2006). Table S1 provides collection details and GenBank accession numbers of all the samples.

\section{Morphological and chemical studies}

Each specimen was studied under a Leica DFC490 dissecting microscope. The secondary metabolites were studied by a $\mathrm{P}$ spot test and thin layer chromatography (TLC) according to standardized procedures described by White and James (1985) and Orange et al. (2001). TLC was performed using solvent systems $\mathrm{A}$ and $\mathrm{C}$ on each specimen included in the phylogeny before DNA extraction.

For the description of new genera, additional material deposited in the Helsinki herbarium was examined. The type specimens of most of the species included in the phylogeny have been studied by T. Ahti. Discussion of morphological characters and species distributions is based on the study of about 500000 specimens in $c a .150$ herbaria worldwide, as well as fieldwork in $c a .50$ countries in North, Middle and South America, Europe, East and South-East Asia, Australasia, and the Pacific. The chemical variation of the species is based on TLC analyses of thousands of specimens over many years by the authors.

\section{Genetic marker selection}

Five markers were selected to infer the Cladoniaceae phylogeny. We included two nuclear ribosomal loci: internal transcribed spacers (ITS rDNA) and intergenic spacers (IGS rDNA); and three protein-coding 
genes: the largest subunit of the RNA polymerase II gene $(R P B I)$ region $\mathrm{A}-\mathrm{F}$, the second largest subunit of RNA polymerase II gene $(R P B 2)$ region 5-7 and the elongation factor-1 $\alpha(E F-1 a)$. Many of the phylogenetic studies on Lecanoromycetes have shown that $R P B 1, R P B 2$ and $E F-1 a$ provide phylogenetic resolution at higher-level taxonomic relationships (Lutzoni et al., 2004; Miadlikowska et al., 2006, 2014; Schoch et al., 2009), whereas the ribosomal markers included here provide information concerning the phylogenetic relationships towards the tips of the phylogenetic tree. ITS rDNA was used by Stenroos et al. (2002a), and we had 235 sequences available from 168 taxa for this marker. Therefore, we decided to use it as well. The $\beta$ tubulin marker, although used by Stenroos et al. (2002a), was discarded, because its PCR success was low and problems associated with paralogy have been found (Begerow et al., 2004). IGS rDNA was selected because it has a large number of polymorphic sites in the genus Cladonia (Pino-Bodas et al., 2013a).

In addition, the nuclear small subunit (nrSSU) was amplified in a selection of samples representing different genera, as this marker has been used in previous phylogenetic studies of Cladoniaceae (Stenroos and DePriest, 1998; Wedin et al., 2000; Stenroos et al., 2002b; Wiklund and Wedin, 2003; Zhou et al., 2006) and sequences from relevant taxa are available in GenBank, many of which were generated by the authors. However, new sequences for the additional markers could not be amplified for these samples, because the extractions were exhausted.

\section{Molecular work}

From each sample, we took a small amount of thallus $(<10 \mathrm{mg})$ for DNA isolation. Before DNA isolation, secondary metabolites were extracted by soaking the samples in acetone, and the resulting solution was used for TLC. A DNeasy Plant Mini Kit (Qiagen, Hilden, Germany) or DNeasy Blood and Tissue Kit (Qiagen) were used to extract DNA, according to the manufacturer's instructions. The DNA was eluted in $150 \mu \mathrm{L}$ of elution buffer provided in the kit. The amplifications of ITS rDNA, $E F-1 \alpha, R P B 1$ and $R P B 2$ were carried out with Ready-to-Go-PCR Beads (GE Healthcare Life Sciences, Little Chalfont, UK) and the amplifications of IGS rDNA were carried out with Biotaq polymerase (Bioline, London, UK). The volume of each reaction was $25 \mu \mathrm{L}$, with $1 \mu \mathrm{L}$ of each primer (10 mM) and $1-5 \mu \mathrm{L}$ of extracted DNA was used, depending on the DNA concentration. The master mix used for Biotaq reactions was the same as described by Pino-Bodas et al. (2013b). The primers used to amplify and sequence the different markers, with their annealing temperature, are listed in Table 1. The amplifications were performed in an Eppendorf
Mastercycler ep gradient S (Westbury, NY, USA). PCR products were purified using the QIAquick gel extraction kit (Qiagen), GFX PCR DNA and Gel Band Purification Kit (GE Healthcare) or ExoSAP-IT (USB Corporation, Cleveland, OH, USA). Sequencing was performed by Macrogen service (macrogen.com), with the same primers as used for the PCR.

\section{Alignments and construction of matrices}

The forward and reverse chromatograms were assembled and edited with the program Sequencher (Gene Codes Corp., Ann Arbor, MI, USA). Each region was aligned separately in BIOEDIT 7.09 (Hall, 1999). IGS rDNA and ITS rDNA were previously aligned in MAFFT (Katoh et al., 2005) using the algorithm G-INS-i, 1PAM/K02 scoring matrix and 0.3 offset value. Subsequently, the alignments were manually improved in BIOEDIT. The SSU rDNA alignment contained numerous introns, which were removed, and only the regions conserved and common for all the sequences were used in the analyses.

For each single gene matrix, a maximum likelihood (ML) analysis in RAxML (Stamatakis et al., 2008) with 500 fast bootstrap replicas was run with the GTRGAMMA model. To identify conflicts among the markers, the clades with more than $75 \%$ bootstrap support (Lutzoni et al., 2004) were examined, using Compat3 (Kauff and Lutzoni, 2002). A few specimens were incongruent among marker trees. Although several biological causes are possible (hybridization, introgression, incomplete lineage sorting, gene duplication), we considered that incongruences were accidental errors generated during laboratory procedures because: (i) they appeared sporadically and they do not affect the phylogeny resolution; (ii) amplifying specimens from tropical areas was problematic (particularly from Réunion); and (iii) $r p b 2$ was amplified by nested PCR and most of the incongruent samples were in this marker. Therefore, we decided to remove these specimens in order to avoid errors in GenBank. Following removal, the ML single marker analyses were repeated with 1000 bootstrap replicas. The final single gene datasets contained the following numbers of sequences: $E F-1 \propto 567$, IGS rDNA 588, ITS rDNA 610, RPBI 415, RPB2 490 (Figs S1-S5) and SSU rDNA 39.

The protein-coding genes did not contain ambiguous positions, but they were numerous in ITS rDNA and IGS rDNA. Different strategies were used to delimit the ambiguous positions in these markers: (i) they were delimited using Gblocks (Talavera and Castresana, 2007) with the less stringent option; (ii) they were manually delimited; or (iii) no positions were removed. ML analyses were run for each strategy and the tree topologies compared among them. All IGS rDNA analyses generated similar topologies with similar 
Table 1

Primers used in the PCR and sequencing of each loci with annealing temperature $\left(T_{\mathrm{m}}\right)$

\begin{tabular}{|c|c|c|c|}
\hline Loci/primers & Primer sequence $\left(5^{\prime}-3^{\prime}\right)$ & $T_{\mathrm{m}}\left({ }^{\circ} \mathrm{C}\right)$ & References \\
\hline \multicolumn{4}{|l|}{$E F-1 a$} \\
\hline CLEF3F & GGCAAAGGCTCCTTCAAGT & 55 & Yahr et al. (2006) \\
\hline CLEF3R & GCCAATACCACCGATCTTGT & 55 & Yahr et al. (2006) \\
\hline \multicolumn{4}{|l|}{ IGS rDNA } \\
\hline IGSf & TAGTGGCCGWTRGCTATCATT & 54 & Wirtz et al. (2008) \\
\hline IGSr & TGCATGGCTTAATCTTTGAG & 54 & Wirtz et al. (2008) \\
\hline \multicolumn{4}{|l|}{ ITS rDNA } \\
\hline ITS1F & CTTGGTCATTTAGAGGAAGTAA & $55-58$ & Gardes and Bruns (1993) \\
\hline ITS4 & TCCTCCGCTTATTGATATGC & $55-58$ & White et al. (1990) \\
\hline nr-SSU-1780 & CTGCGGAAGGATCATTAATGAG & 55 & Piercey-Normore and DePriest (2001) \\
\hline nr-LSU-0012 & AGTTCAGCGGGTATCCCT & 55 & Piercey-Normore and DePriest (2001) \\
\hline ITS1LM & GAACCTGCGGAAGGATCATT & $55-58$ & Myllys et al. (1999) \\
\hline ITS2KL & TCCTCCGCTTATTGATATGC & $55-58$ & Lohtander et al. (1998) \\
\hline \multicolumn{4}{|c|}{ 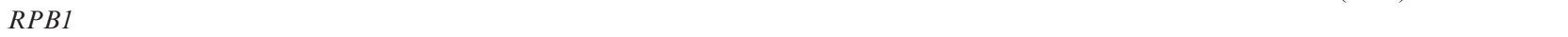 } \\
\hline RPB1Af & GARTGYCCDGGDCAYTTYGG & $54-52$ & Stiller and Hall (1997) \\
\hline $\mathrm{RPB} 1 \mathrm{Cr}$ & CCNGCDATNTCRTTRTCCATRT & $54-52$ & Stiller and Hall (1997) \\
\hline \multicolumn{4}{|c|}{ 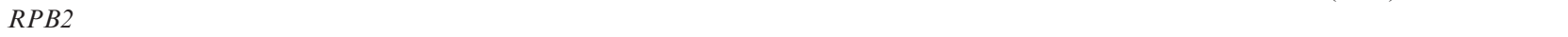 } \\
\hline RPB2-5F & GAYGAYNGWGATCAYTTYGG & 52 & Liu et al. (1999) \\
\hline RPB2-7R & CCCATRGCTTGYTTRCCCAT & 52 & Liu et al. (1999) \\
\hline CLRPB25F & CTGTTTCGAACGCTGTTTCA & 52 & Yahr et al. (2006) \\
\hline CLRPB27R & CGCATCCACGTATTCAACAA & 52 & Yahr et al. (2006) \\
\hline RPB2dRaq & GCTGCTAAGTCTACCAT & 52 & Pino-Bodas et al. (2010) \\
\hline RPB2rRaq & ATCATGCTTGGAATCTC & 52 & Pino-Bodas et al. (2010) \\
\hline SSU rDNA & & $53-55$ & \\
\hline SSU0072 & CATGTCTAAGTTTAAGCAA & $53-55$ & Gargas and DePriest (1996) \\
\hline SSU0852 & CGTCCCTATTAATCATTACG & $53-55$ & Gargas and DePriest (1996) \\
\hline SSU0819 & GAATAATAGAATAGGACG & $53-55$ & Gargas and DePriest (1996) \\
\hline SSU1750 & TAAAAGTCGTAACAAGGTITCCGTAGG & $53-55$ & Gargas and DePriest (1996) \\
\hline SSU0402 & CCGGAGAAGGAGCCTGAGAAAC & $53-55$ & Gargas and DePriest (1996) \\
\hline SSU1293 & AATTAAGCAGACAAATCACT & $53-55$ & Gargas and DePriest (1996) \\
\hline
\end{tabular}

support values. However, the topology of the ITS rDNA tree from Gblocks was different from the other ITS rDNA analyses and other marker analyses. For instance, Metus conglometarus fell within Cladonia, while in the analyses of other markers $(E F-1 \alpha, R P B 1$ and $R P B 2$ ) this genus was distantly related to Cladonia. Based on these results we decided to delimit the ambiguities manually for ITS rDNA and IGS rDNA datasets. Only a few sequences were incongruent among the single gene analyses (apparent contaminants or code errors). These sequences were removed and the matrices were then concatenated.

SequenceMatrix 1.78 (Vaidya et al., 2011) was used to concatenate the single gene matrices. Following the supermatrix approach (Miadlikowska et al., 2006), four concatenated datasets were generated: (i) 5 gene dataset, containing only specimens with sequences from 5 markers; (ii) $5+4$ gene dataset, containing only specimens with sequences from at least 4 markers; (iii) $5+4+3$ gene dataset, containing only specimens with sequences from at least 3 markers; and (iv) $5+4+3+2$ gene dataset, containing only specimens with sequences from at least 2 markers. Finally, we built a concatenated matrix of all sequences
$(5+4+3+2+1$ gene) to maximize the number of terminal taxa included.

\section{Phylogenetic analyses}

Maximum parsimony (MP), ML and Bayesian analyses were conducted on each of the four concatenated datasets (5 gene, $5+4$ gene, $5+4+3$ gene and $5+4+3+2$ gene) and on the SSU rDNA dataset. In addition, the two largest concatenated datasets $(5+4+3+2$ gene and $5+4+3+2+1$ gene) were analysed by MP using TNT (Goloboff et al., 2008; Goloboff and Catalano, 2016). Currently this program provides the most efficient algorithms for the analyses of large datasets (>200 terminal taxa). The inclusion of terminals with only one sequence was intended to determine their effect on the results. Although "pathological behaviour" (Wheeler, 2012) of missing entries is well known (e.g. Nixon and Davis, 1991; Platnick et al., 1991) several studies (e.g. Wolsan and Sato, 2010; Lehtonen, 2011) suggest that despite the deleterious effect of missing information, analyses of even sparse matrices is worthwhile. Other MP analyses (5 gene, $5+4$ gene, $5+4+3$ gene and $5+4+3+2$ 
gene) were performed using PAUP* version 4.0.b.10 (Swofford, 2003). Heuristic searches were performed with 1000 random taxon-addition replicates using treebisection-reconnection (TBR) branch swapping and the MulTrees option, equally weighted characters, and gaps treated as missing data. Branch support values were calculated using bootstrap with 10000 replicates and the "fast option".

Analyses $(5+4+3+2$ gene and $5+4+3+2+1$ gene) using TNT were performed with the search settings used in Wheeler et al. (2017). Initially, parsimony-uninformative characters were removed from the matrix using the "mop uninformative characters" function of the program Winclada (Nixon, 1999). This resulted in a matrix of 606 terminals and 1723 characters for the $5+4+3+2$ dataset, and 657 terminals and 1736 characters for the $5+4+3+2+1$ dataset. Searches were initiated with ten replicates of RAS (random addition sequence) + TBR (tree-bisection-reconnection) branch swapping, and this was performed 30 times to get a large pool of diverse trees that were already optimized rather than a sample of random trees possibly far from the optimal solution. After this, each set of 10-30 trees (trees saved per replicate 1,2 or 3 ) obtained were subjected to the new technology search composed of sectorial search (random and mixed sectorial searches) plus tree-drifting (Goloboff, 1999), with default settings. Finally, all trees were combined and subjected to tree-fusing (Goloboff, 1999), again using default settings of the program. Parsimony jackknife (Farris et al., 1996) support values were calculated using TNT with default settings of the traditional search with 1000 replicates.

Initially, the protein-coding gene datasets were analysed by ML analyses with two different partition schemes: (i) each marker was considered as a single partition; and (ii) each codon position was considered as a separate partition. The results of the two strategies were similar in all cases, so we decided to analyse the four concatenated datasets considering each marker as a separate partition in ML and Bayesian analyses, in order to reduce the computation time. ML analyses were run using the same conditions as in the ML single marker analyses with 1000 bootstrapped replicates. The Akaike information criterion in MrModeltest (Nylander, 2004) was used to determine the best fit nucleotide substitution models for each marker. The models selected were: GTR $+\mathrm{I}+\mathrm{G}$ for $E F-1 a$, IGS rDNA, ITS rDNA, SSU rDNA and $R P B 1$; and $\mathrm{SYM}+\mathrm{I}+\mathrm{G}$ for $R P B 2$. These models were used to run Bayesian analyses. The Bayesian analyses were run using MrBayes 3.2 (Ronquist et al., 2012) on the CIPRES Science Gateway website portal (Miller et al., 2010). Posterior probabilities were approximated by sampling trees using Markov chain Monte Carlo (MCMC) analysis. The posterior probability (pp) of each branch was calculated by counting the frequency of trees visited during MCMC analysis. We executed two simultaneous runs with 30000000 generations, employing one cold and three hot chains, with the temperature of the chains set to 0.05 . Prior branch length was set to "brlenspr=unconstrained:exp(50)". Default values were left for the remaining priors. Every 1000th tree was saved into a file. The convergence between the runs was assessed with standard deviation of split frequencies $<0.01$. AWTY (Nylander et al., 2008) was used to determine when the chains reached the stationary stage. The first $50 \%$ of generations were deleted as the "burn-in" of the chain. The $50 \%$ majority-rule consensus tree was calculated using the "sumt" command of MrBayes from the post-burn-in trees.

Bootstrap and parsimony jackknife values $\geq 70 \%$, and $p p$ values $\geq 0.95$ were used as thresholds of "adequate" support for the nodes according to previous studies (Alfaro et al., 2003; Soltis and Soltis, 2003; Simmons et al., 2004).

\section{Results}

Altogether we generated 2118 new sequences, 530 of $E F-1 \alpha, 407$ of $R P B 1,410$ of $R P B 2$, ten of SSU rDNA, 466 of IGS rDNA and 295 of ITS rDNA. The ML analyses of single marker matrices are presented in Figs S1-S5. The marker with the largest number of informative characters was ITS rDNA (430) followed by $R P B 1$ (399), RPB2 (351), IGS rDNA (290) and $E F-1 \alpha$ (266). However, the marker with the highest percentage of clades with bootstrap values $\geq 70 \%$, and pp $\geq 0.95(54.12 \%)$ was $R P B 1$ followed by $R P B 2$ $(50.308 \%)$, ITS rDNA $(43 \%)$, IGS rDNA $(41.36 \%)$ and $E F-1 \alpha(41.134 \%)$.

Table 2 summarizes the different values of the analyses for the concatenated datasets and SSU rDNA. MP (PAUP), ML and Bayesian analyses on the same concatenated dataset (5 gene to $5+4+3+2$ gene datasets) all yielded the same tree topology with the same main clades. MP (TNT) analyses also yielded the same general tree topology $(5+4+3+2$ gene and $5+4+3+2+1$ gene datasets). MP (TNT) of the $5+4+3+2$ gene dataset yielded a shorter and thus more optimal tree comparable to PAUP analysis $(5+4+3+2$ gene). Figure 1 shows a cartoon tree with the main clades. Figure 2 shows the $50 \%$ majority-rule compromise tree from the Bayesian analysis for the $5+4+3+2$ gene dataset.

The family Cladoniaceae was supported in all analyses with bootstrap values $\geq 70 \%$, and $\mathrm{pp} \geq 0.95$, or Jackknife values $\geq 70$. Thysanothecium and Notocladonia were nested within Cladia s.l. (Fig. 2). The following four clades were recovered with bootstrap values $\geq 70 \%$ 
Table 2

Features of the datasets used in this study and phylogenetic information from the analyses

\begin{tabular}{|c|c|c|c|c|c|c|c|}
\hline & Taxa & $N$ & Char (bp) & PI & Length & -Lnl Bayesian & $-\mathrm{Lnl} \mathrm{ML}$ \\
\hline 5 gene & 193 & 346 & 3336 & 1555 & 12269 & 69399.15 & 74168.35 \\
\hline $5+4$ gene & 262 & 517 & 3369 & 1677 & 16122 & 88903.26 & 95556.84 \\
\hline $5+4+3$ gene & 285 & 572 & 3387 & 1701 & 17054 & 94149.94 & 101016.45 \\
\hline $5+4+3+2$ gene & 302 & 606 & 3390 & 1723 & $\begin{array}{l}17624^{*} \\
17600^{\dagger}\end{array}$ & 104690.5 & 103910.03 \\
\hline $5+4+3+2+1$ gene & 304 & 657 & & 1736 & $18068^{\dagger}$ & - & - \\
\hline nrSSU & 28 & 40 & 1694 & 66 & 221 & 5322.64 & 5824.49 \\
\hline
\end{tabular}

$N$, number of sequences included in each dataset; Char, total number of positions included in each dataset; PI, parsimony-informative positions; Length, number of steps of the most parsimonious trees.

*MP analysis run in PAUP.

${ }^{\dagger} \mathrm{MP}$ analysis run in TNT.

and $\mathrm{pp} \geq 0.95$, or jackknife inside Cladia: (i) a clade containing Cladia sullivanii and C. fuliginosa; (ii) a clade containing Cladia muelleri, C. beaugleholei, Thysanothecium scutellatum and Notocladonia cochleata; (iii) a clade containing the Cladia aggregata complex with morphologically similar species including C. aggregata, C. cryptica, C. deformis, C. dumicola, C. gorgonea, C. inflata, C. moniliformis, C. neocaledonica, C. terebrata and C. schizopora; and (iv) a clade containing Cladia retipora and $C$. corallaizon. Pilophorus was the sister group to Cladia s.l. in most of the analyses, but this relationship was not as strongly supported, with bootstrap values $<70 \%$ and $\mathrm{pp}<0.95$. In the TNT analysis $(5+4+3+2$ gene) Pilophorus resolved as sister to the rest of the tree, namely Cladia s.l. plus Cladia's sister clade including Pycnothelia, Carassea, Metus, and Cladonia, with the jackknife value $\geq 70 \%$. Finally, in the $5+4+3+2+1$ gene tree the placement of Pilophorus could not be resolved because of the absence of resolution in that part of the tree. The clade comprising Pycnothelia, Carassea and Metus was monophyletic, supported with bootstrap values $\geq 70 \%$ and $\mathrm{pp} \geq 0.95$, or jackknife $\geq 70 \%$. The clade was sister to Cladonia in all analyses, and C. wainioi was sister to the latter. Finally, and importantly, Cladonia was supported with bootstrap values $\geq 70 \%$ and $\mathrm{pp} \geq 0.95$, or jackknife value $\geq 70 \%$ in all concatenated analyses.

In most of the analyses, 13 major groups inside Cladonia, referred to as Clades, were resolved with bootstrap values $\geq 70 \%$ and $\mathrm{pp} \geq 0.95$ (Fig. 1 and Table 3 ). In the TNT $5+4+3+2$ gene analysis only Clade Unciales received jackknife support $<70 \%$ (the value was $65 \%$ ), while the $5+4+3+2+1$ gene analysis left $C$. steyermarkii in a large unresolved "polytomy"; the rest of Clade Unciales received jackknife support $<70$.

The Clades are reported in Fig. 1 using the following names (many are the earliest names at the formal sectional level, while some have never been used nomenclaturally): Clade Cladonia, Clade Borya, Clade Unciales, Clade Crustaceae, Clade Arbuscula, Clade
Amaurocraeae, Clade Ochroleucae, Clade Divaricatae, Clade Perviae, Clade Erythrocarpae, Clade Delavaya, Clade Impexae and Clade Wainioa.

Subclades were defined in some of the larger Clades: Clade Erythrocarpae (three Subclades), and Clade Cladonia, which contains most of the species, was divided into 11 Subclades (Figs 1 and 2). The bootstrap, posterior probability, and jackknife values of the Subclades in the different analyses are summarized in Table 3. However, only in the five gene dataset analyses did the relationships between the Subclades inside Cladonia receive bootstrap support $\geq 70 \%$ and $\mathrm{pp} \geq 0.95$ (Fig. 1). Those recognized are as follows: Subclades Miniatae, Incrassatae and Subclaucescentes in Clade Erythrocarpae, and Subclades Firmae, Rangiformes, Macropus, Graciles, Foliaceae, Vesculae, Cladonia, Helopodium, Apodocarpae, Ascyphiferae and Callosae in Clade Cladonia. The Clades, Subclades, and their relationships can be seen in Fig. 1.

The consensus tree obtained from the TNT $5+4+3+2+1$ gene analysis has a similar topology to the other analyses but it lost resolution in the backbone of the tree and within some of the Clades (Fig. S6). Several relationships at the species level were also poorly resolved, particularly within the Clades Cladonia and Perviae.

Figure 3 shows the $50 \%$ majority-rule tree from the Bayesian analysis for the nrSSU dataset. Cladoniaceae was found to be monophyletic as a family (bootstrap support $<70 \%$ and pp $<0.95$ ). One recovered clade contained Cetradonia, Gymnoderma, Pycnothelia and Carassea (81\% ML bootstrap support, 76\% MP bootstrap and pp of 1.0); this clade was sister to Cladonia, but with a lower support value. Cetradonia and Gymnoderma together appeared as the sister group, with bootstrap support values of $93 \%$ (ML) and $91 \%(\mathrm{MP})$, and pp of 0.96. Carassea was sister to Pycnothelia. Cladonia was monophyletic, with $C$. wainioi as the earliest diverging lineage in the genus. Pilophorus was monophyletic only in the ML analysis, but $P$. strumaticus joined the 


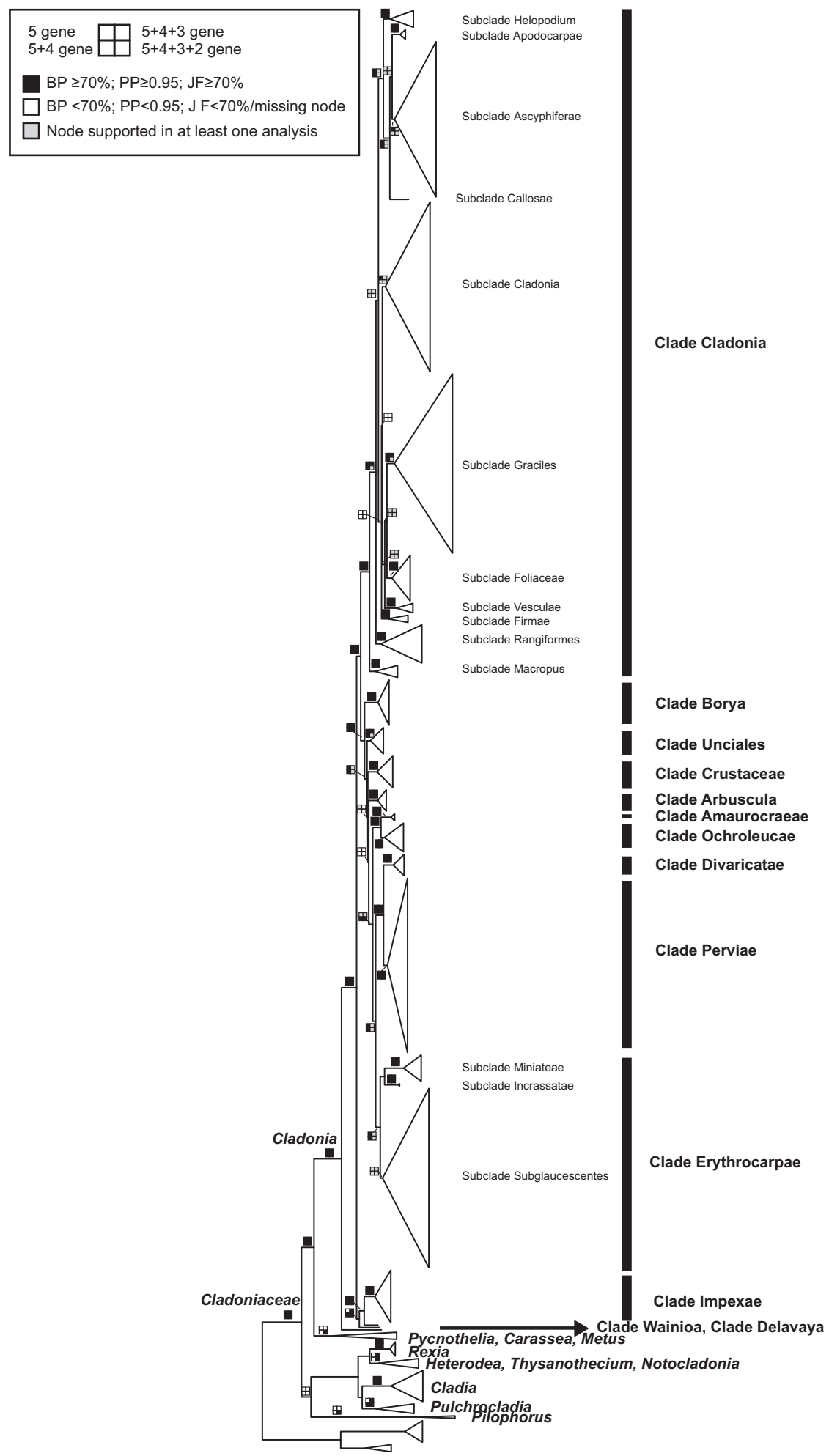

Fig. 1. Cartoon tree $(5+4+3+2$ gene) representing the phylogenetic relationships within Cladoniaceae. It is based on a five-locus $50 \%$ majority-rule consensus tree from the Bayesian analysis. Boxes associated with each node indicate support in the analyses of the different datasets: black, bootstrap values $\geq 70 \%$, pp $\geq 0.95$ and jackknife value $\geq 70 \%$; white, absence of node or bootstrap value $<70 \%$ or pp $<0.95 \%$ or jackknife value $<70 \%$; grey, node supported only in some of the analyses of the dataset. 


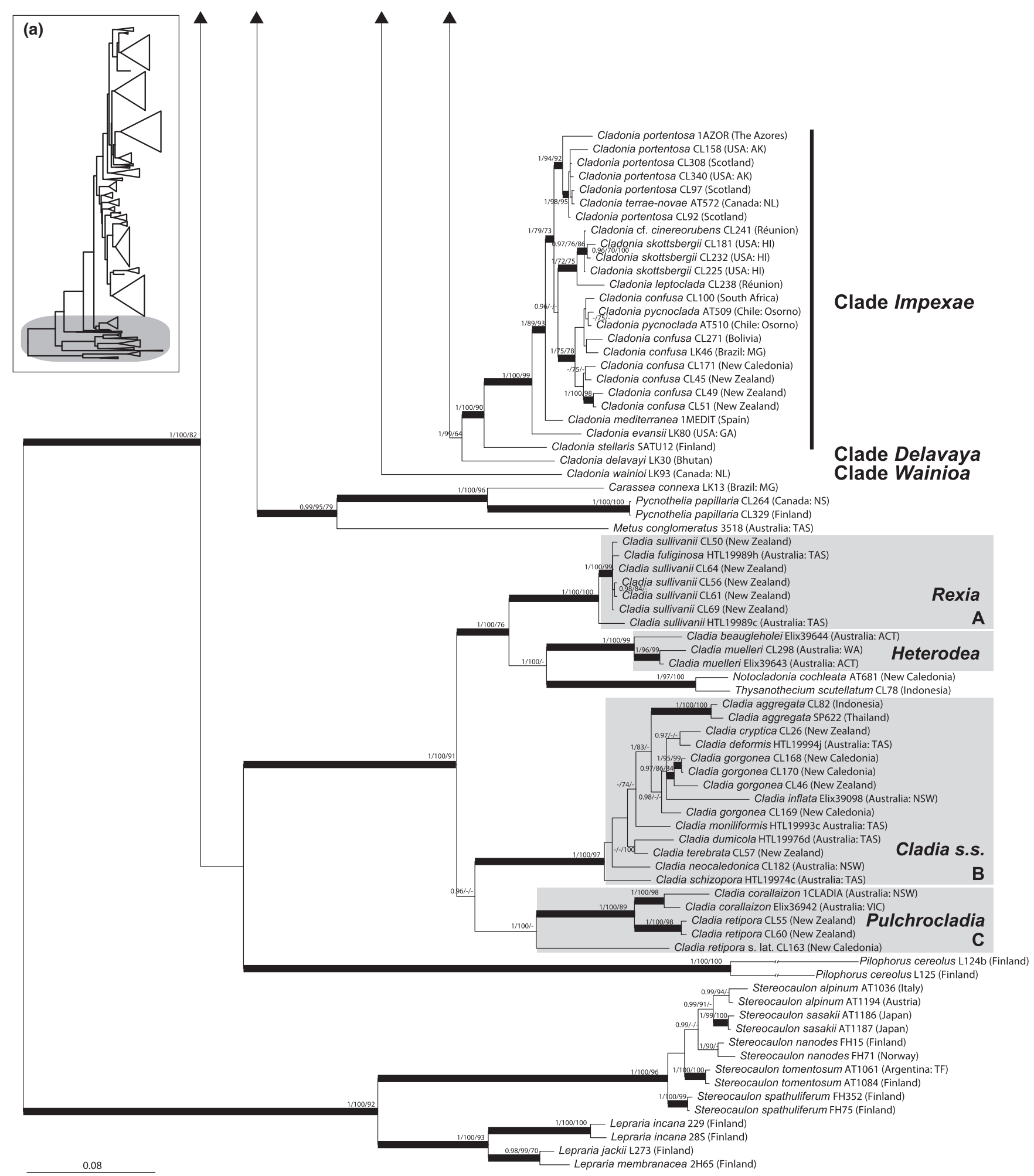

Fig. 2. Phylogeny of Cladoniaceae estimated with five loci (ITS rDNA, IGS rDNA, $E F-1 \alpha$, RPB1 and $R P B 2$ ). This is the $50 \%$ majority-rule consensus tree of Bayesian analysis based on the $5+4+3+2$ dataset. Branches supported with posterior probability $\geq 0.95$ and bootstrap $\geq 70 \%$ are indicated in bold. Numbers on the branches are the posterior probability values from Bayesian analysis/bootstrap values from ML/ bootstrap values from MP analysis. After taxon name and DNA code, the geographical origin of specimens is indicated. Abbreviations correspond to states/provinces of the US, Canada, Mexico, Brazil, Argentina and Australia; RS = Russia. 

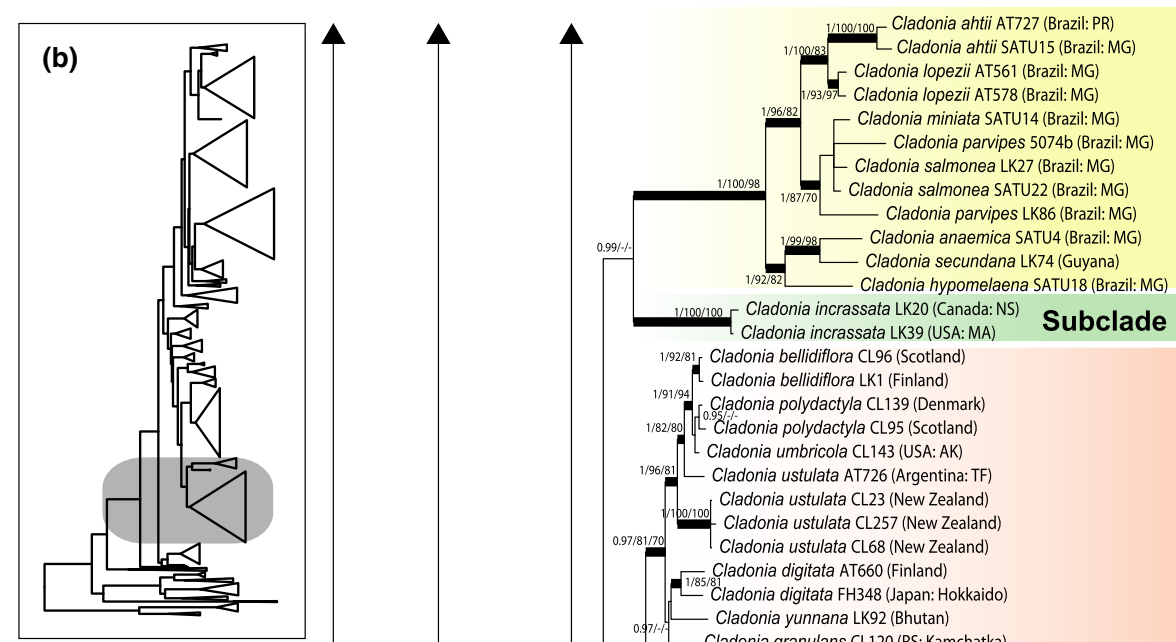

Cladonia incrassata LK20 (Canada: NS)

Cladonia incrassata LK39 (USA: MA)

Subclade Incrassatae 1/92/81 Cladonia bellidiflora CL96 (Scotlan

L Cladonia bellidiflora LK1 (Finland)

1/91/94 Cladonia polydactyla CL139 (Denmark)

1/82/80] C. Cladonia polydactyla CL95 (Scotland)

Cladonia umbricola CL143 (USA: AK)

1/96/81 $\angle$ Cladonia ustulata AT726 (Argentina: TF)

Cladonia ustulata CL23 (New Zealand)

Cladonia ustulata CL257 (New Zealand)

Cladonia ustulata CL68 (New Zealand)

$\sqrt{1 / 85581}$ Cladonia digitata AT660 (Finland)

1/85/81 Cladonia digitata FH348 (Japan: Hokkaido)

7 Cl- Cladonia yunnana LK92 (Bhutan)

_ Cladonia granulans CL120 (RS: Kamchatka)

1/83/88 _ Cladonia camerunensis CL102 (Cameroon) Cladonia cristatella LK17 (Canada: NS)

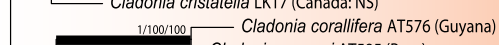

Cladonia prancei AT585 (Peru)

1/100/99 Cladonia leporina CL199 (USA: GA) 1/100/99 Cladonia leporina CLig9 (USA: GA)

0.991877) 1/100/99L Cladonia leporina LK84 (USA: GA)

$\begin{aligned} 1 / 100 / 100 & \text { Cladonia ravenelii CL200 (USA: FL) } \\ \text { Cl/100/100 } & \text { Cladonia ravenelii LK87 (USA: GA) }\end{aligned}$

$\underset{1 / 100 \%}{ }$

L Cladonia ravenelii CL201 (USA:
1/100/100 Cladonia hypoxantha CL203 (USA: SC)

Cladonia hypoxantha CL235 (USA: SC)

Cladonia longisquama CL154 (Seychelles)

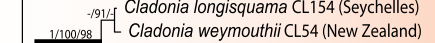

- Cladonia macilenta s. lat. CL165 (New Caledonia)

- Cladonia macilenta s. lat. CL165 (New Caledon Cladonia vulcani CL137 (RS: Kamchatka)

0.98/933. Cladonia floerkeana AT728 (Taiwan)

1.835 - Cladonia macilenta s. lat. CL17 (New Zealand)

L Cladonia floerkeana CL85 (USA:NY)

75/195 Cladonia macilenta 1GLAU (Spain)

Cladonia macilenta LK54 (Canada: NS)

Cladonia macilenta CL9 (New Zealand)
Cladienta

Cladonia straminea LK3 (Finland)

181 Cladonia didyma s. lat. CL79 (Indonesia)

Cladonia meridensis $\mathrm{CL} 295$ (Bolivia)

- Cladonia meridensis CL265 (Bolivia)

1/866- Cladonia microscypha CL320 (Bolivia)

Cladonia mis

Cladonia leprocephala CL266 (Bolivia)

Cladonia leprocephala CL266 (Bolivia)

Cladonia leprocephala CL267 (Bolivia)

Cladonia leprocephala CL289 (Bolivia)

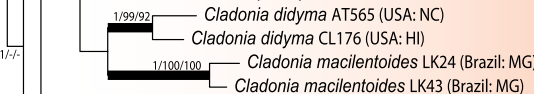

Cladonia coccifera LK28 (Bhutan)

Cladonia coccifera LK26 (Bhutan)

Cladonia coccifera LK55 (Canada: NL)

Cladonia diversa CL231 (Greece)

Cladonia coccifera s. lat. SATU11 (Iceland)

- Cladonia coccifera LK2 (Finland)

Cladonia insignis CL152 (Réunion)

donia sulphurina LK77 (Greenland)

- Cladonia lepidophora AT504 (Chile: Valdivia)

- Cladonia lepidophora AT504 (Chile: Valdivia)

Cladonia lepidophora CL89 (Chile: Magallanes)

Cladonia diversa CL290 (Bolivia)

99:47/802 Cladonia borealis LK4 (Finland)

L Cladonia borealis LK53 (Iceland)

Cladonia pleurota CL27 (New Zealand)

$1 / 88$

Cladonia pleurota CL303

Cladonia pleurota AT639 (USA: GA)

Cladonia pleurota AT639 (USA: GA)

1/99//65. Cladonia pleurota AT705 (Australia)

Cladonia pleurota AT536 (Canada: NS)
Cladonia pleurota AT587 (Mexico: VC)

Cladonia coccifera s. lat. CL189 (Réunion) Cladonia deformis 1905 (USA: NH)

Cladonia deformis CL305 (RS: Murmansk)

Cladonia deformis CL337 (Finland)

Cladonia deformis 1258 (Finand)

1/83/96L Cladonia deformis CL260 (Finland)

Fig. 2. Continued 

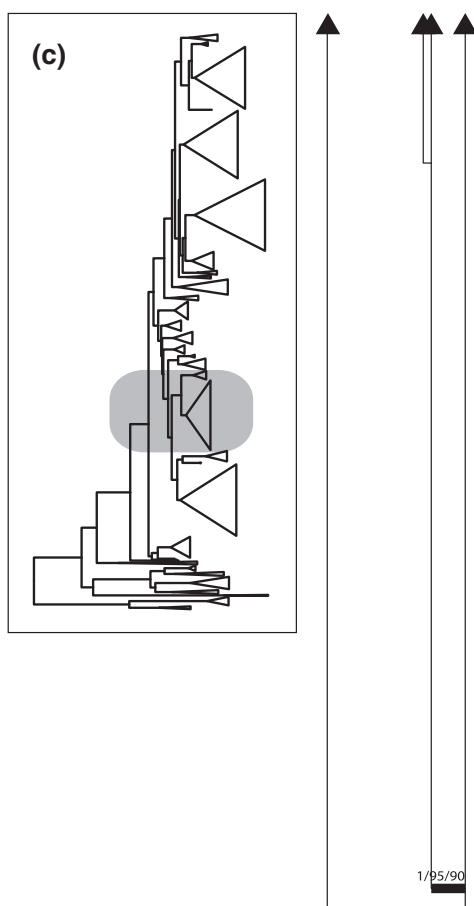

4 1/99/96 Cladonia albofuscescens SATU5 (Brazil: MG)

- Cladonia albofuscescens SATU9 (Brazil: MG) 1/100/97 [ Cladonia minarum SATU7 (Brazil: MG)

LCladonia divaricata SATU10 (Brazil: MG)

1/92/78 Cladonia variegata AT601 (Guyana)

-778/1/100/100 - Cladonia consimilis SATU1 (Brazil: MG)

- Cladonia consimilis SATU2 (Brazil: MG)

1/100/100 Cladonia fleigiae AT710 (Brazil: MG)

Cladonia bahiana AT715 (Brazil: BA)

Cladonia bahiana AT716 (Brazil: MG)

1/93/83 - Cladonia atlantica LK10 (Canada: NL)

1/93/83 - Cladonia atlantica LK10 (Canada: NL)
1/71/- Cladonia atlantica LK42 (USA: MA)

Cladonia crispata s. lat. CL229 (Greece)
Cladonia atlantica LK42 (USA: MA)

Cladonia granulosa CL355 (Costa Rica)

$0.97 /-1$

0.97/t- Cladonia granulosa CL292 (Bolivia)

198/-796/80 — Cladonia crispatula AT597 (Brazil: MG)

1/100/87 L Cladonia hians AT583 (Guyana)

Cladonia polystomata AT595 (Guyana)
Cladonia hians AT583 (Guyana)

0,97 Cladonia polystomata AT595 (Guyana)
$0.100 / 96$
Cladonia subdelicatula CL136 (Réunion)

1.99/- Cladonia subdelicatula CL352 (Costa Rica)

Cladonia dilleniana CL312 (Cuba)

1/97/87_ Cladonia subsetacea CL141 (USA: FL)

1/197/87 - Cladonia subsetacea CL141 (USA: FL)

1/100/100 L Cladonia subsetacea DW19 (USA:

0.95/-1- Cladonia beaumontii AT633 (USA. Cladonia cenotea LK62 (Finland)

[1/-l- Cladonia cenotea LK81 (Argentina: TF)

(1)/99: Cladonia cenotea 1CENO (Denmark)

Cladonia glauca CL214 (Greece)

Cladonia cenotea LK9 (Canada: NL)

0.99/23/ádonia squamosa SATU16 (Sweden)

0.991/2. L L Cladonia varians CL144 (Réunion)

1/100/96_ Cladonia rugulosa AT577 (Guyana)

Cladonia vareschii LK85 (Guyana)

Cladonia poroscypha CL105 (USA: OR)

Cladonia capitellata CL184 (Australia: WA)

Cladonia subsubulata CL19 (New Zealand)

Cladonia subsubulata 1AUERI (Chile: Chiloé)

1/901/99 Cladonia subsubulata AT725 (Argentina: TF)

Cladonia subsubulata AT744 (Falkland Is.)

- Cladonia subsubulata AT745 (Falkland Is)

1/95/- - Cladonia capitellata CL21 (New Zealand)

Cladonia capitellata CL66 (New Zealand)

- Cladonia capitellata CL53 (New Zealand)

Cladonia capitellata CL73 (New Zealand)

Cladonia subsubulata CL62 (New Zealand) Cladonia subsubulata CL52 (New Zealand)

Cladonia subsubulata CL72 (New Zealand)

- Cl/86/5 donia subsubulata CL76 (New Zealand)

- Cladonia subsubulata CL74 (New Zealand)

L Cladonia subsubulata CL65 (New Zealand)

1.17- Cladonia capitellata CL39 (New Zealand)

- Cladonia capitellata CL39 (New Zealand)

- Cladonia pertricosa AT737 (Australia: NSW)

- Cladonia crispata s. lat. CL81 (Indonesia)

- Cladonia crispata AT648 (Finland)

Cladonia parasitica 1PARAS (Spain)

$100 / 92$ Cladonia parasitica 2PARA (Germany)

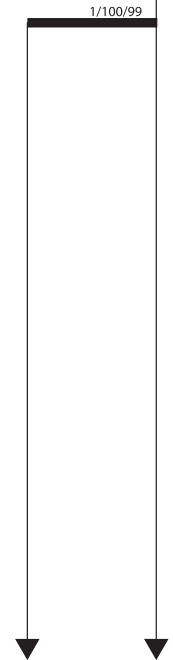

1/97/99 _ Cladonia candelabrum s. lat. CL106 (Madagascar)

1/91/85 L Cladonia candelabrum DW21 (Réunion)

190 L Cladonia sp. Cl 215 (Réunion)

1/99/85 Cladonia hedbergii AT682 (Zimbabwe)

Cladonia gigantea CL160 (Madagascar)

100/98 Cladonia pachyclados DW20 (Réunion)

Cladonia gigantea s. lat. CL240 (Réunion)

100 Cladonia medusina CL161 (New Zealand)

1/97/8T Cladonia medusina CL162 (New Zealand)

1/72/- - Cladonia sp. LK61 (Finland)

0.95/-l-Cladonia subfurcata LK21 (Finland)

Cladonia hondoensis CL103 (Japan: Hokkaido)

0.88/83/72 Cladonia rigida CL58 (New Zealand)

1/80/- Cladonia rigida CL15 (New Zealand)

119p/100 Cladonia rigida CL192 (Australia: WA)

Cladonia rigida CL75 (New Zealand)

Cladonia sp. AT743 (Australia)

Cladonia metaminiata LK14 (Brazil: MG)

1/100/100 Cladonia piedadensis SATU23 (Brazil: MG) Cladonia santensis CL133 (USA: NJ)

Cladonia strepsilis AT537 (Finland)

100/100 [ Cladonia strepsilis LK18 (C Canada:NS)

Cladonia strepsilis CL91 (Scotland)

\section{Clade Divaricatae}

Clade Perviae

Fig. 2. Continued 


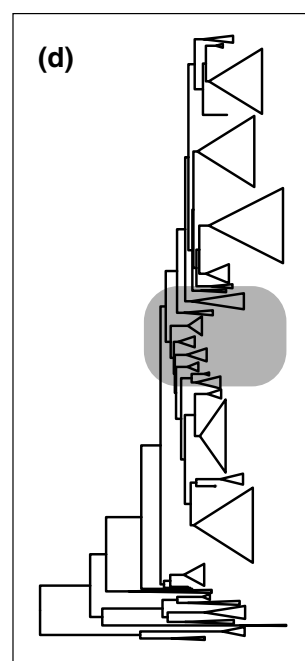

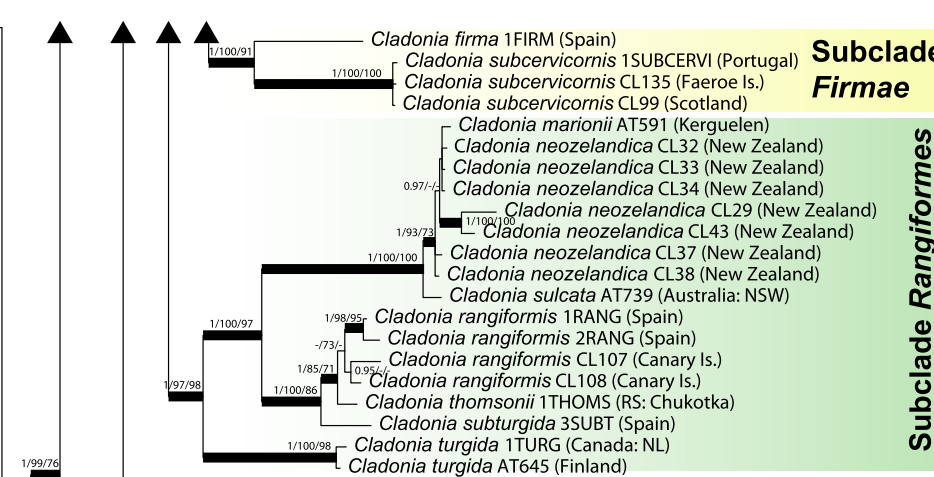

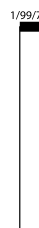

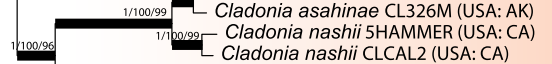
$1 / 10100 \quad$ Cladonia nashii CLCAL2 (USA: CA)
Cladonia brevis CL114 (USA:PA) Cladonia macrophylla CL328

1/100/100 - Cladonia boryi LK50 (Canada: NL)

Cladonia boryi LK6 (Canada: NS)

Cladonia zopfii FH344 (Portugal)

1/100/96 [1/ Clag9donia zopfii SATU37 (The Netherlands)

Cladonia nipponica CL213 (USA: AK)

Cladonia nipponica FH338 (RS: Primorye)

190/922 Cladonia caroliniana AT636 (USA: NS)
00/92 Cladonia caroliniana CL226 (USA: NC)

Cladonia dimorphoclada AT635 (USA: SC)

Cladonia pachycladodes DW18 (USA: FL)

Cladonia kanewskii CL244 (RS: Kamchat

o/100 l0.9877/8 donia kanewskii LK52 (USA: AK)

\$1/3386 Cladonia kanewskii CL245 (RS: Kamchatka)

Cladonia kanewskii s. lat. DW22 (RS: Buryatia)

100 - Cladonia stenroosiae LK25 (Brazil:MG)

- Cladonia substellata SATU21 (Brazil:MG)

1/1960ladonia substellata SATU6 (Brazil: MG)

- Cladonia subreticulata SATU3 (Brazil: MG)

175/89. Cladonia uncialis subsp. biuncialis CL210 (Greece)

Cladonia uncialis subsp. biuncialis DW12 (Iceland)

Cladonia uncialis subsp. biuncialis DW1 (France)

Cladonia uncialis subsp. biuncialis DW10 (Finland)

Cladonia uncialis subsp. biuncialis $\mathrm{FH} 233$ (Canada: NL)

Cladonia uncialis subsp. uncialis FH328 (Japan: Honshu)

-Cl-celadonia uncialis subsp. uncialis CL204 (Finland)

1100/100 Cladonia uncialis subsp. uncialis CL246 (Canada: NS)

'Cladonia uncialis subsp. uncialis FH234 (Canada: NL)

1/89-- Cladonia uncialis subsp. uncialis FH332 (RS: Kamchatka)

Clonia uncialis subsp. uncialis CL233

Subclade Macropus

\section{Clade Cladonia}

Cladonia steyermarkii LK89 (Guyana)

1/100/95 1 - Cladonia subtenuis AT571 (Canada: NS)

1/96/88 Cladonia subtenuis AT637 (USA: GA)

Cladonia ciliata var tenuis AT842 (Portugal)

Cladonia ciliata var ciliata AT843 (Ireland)

Cladonia ciliata var ciliata CL 93 (Scotland)

Cladonia argentea LK66 (Guyana)

o.pp/74/83 Cladonia rotundata AT603 (Guyana)

L Cladonia sprucei AT599 (Guyana)

Cladonia rangiferina subsp. abbayesii AT594 (Guyana)
Clade Borya

Clade Borya
Clade Unciales

Clade Crustaceae

Clade Arbuscula

Clade Amaurocraeae

Clade Ochroleucae

Fig. 2. Continued

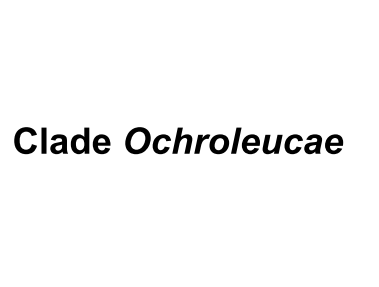




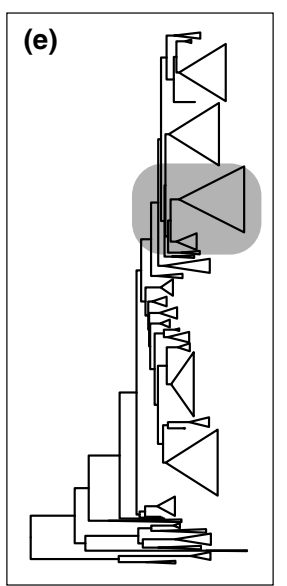

C Cladonia alinii CL111 (RS: Primorye)

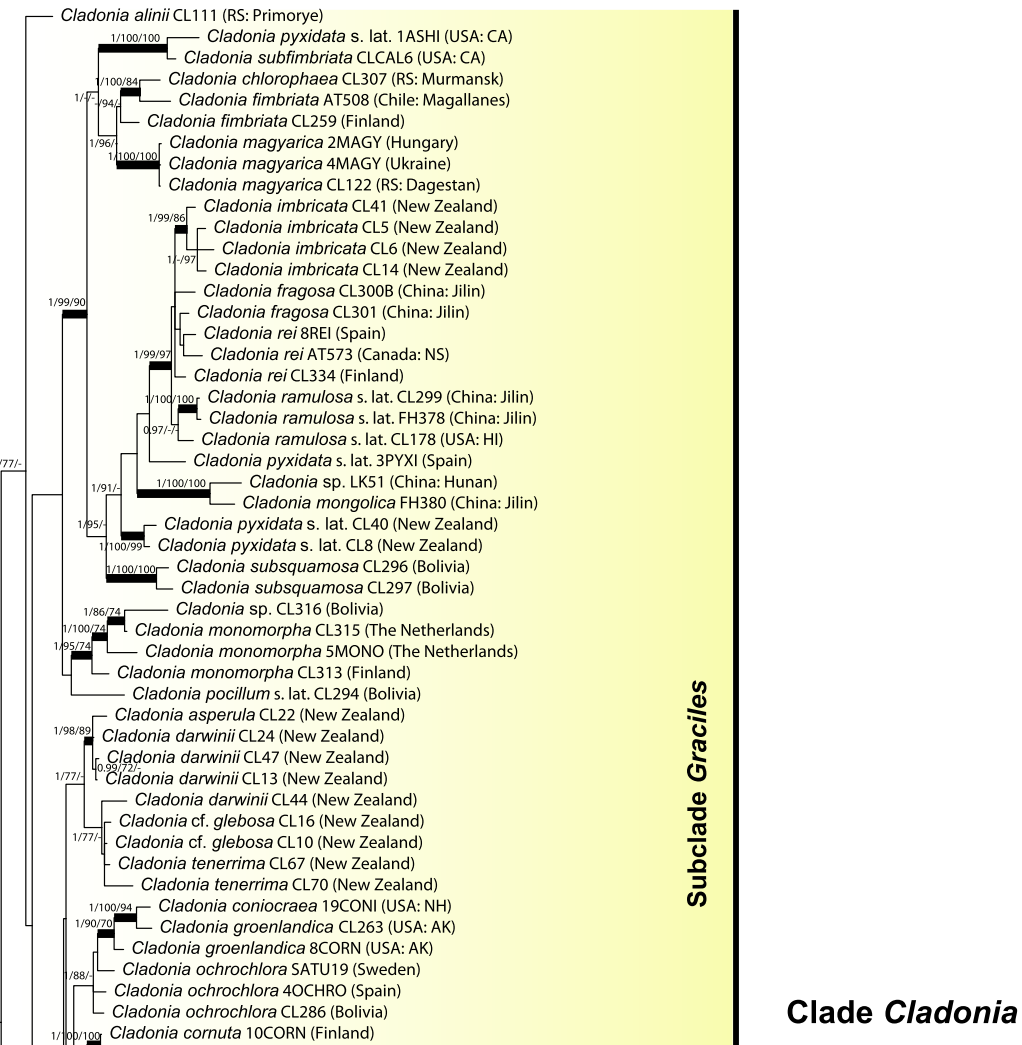

Clade Cladonia

Fig. 2. Continued 

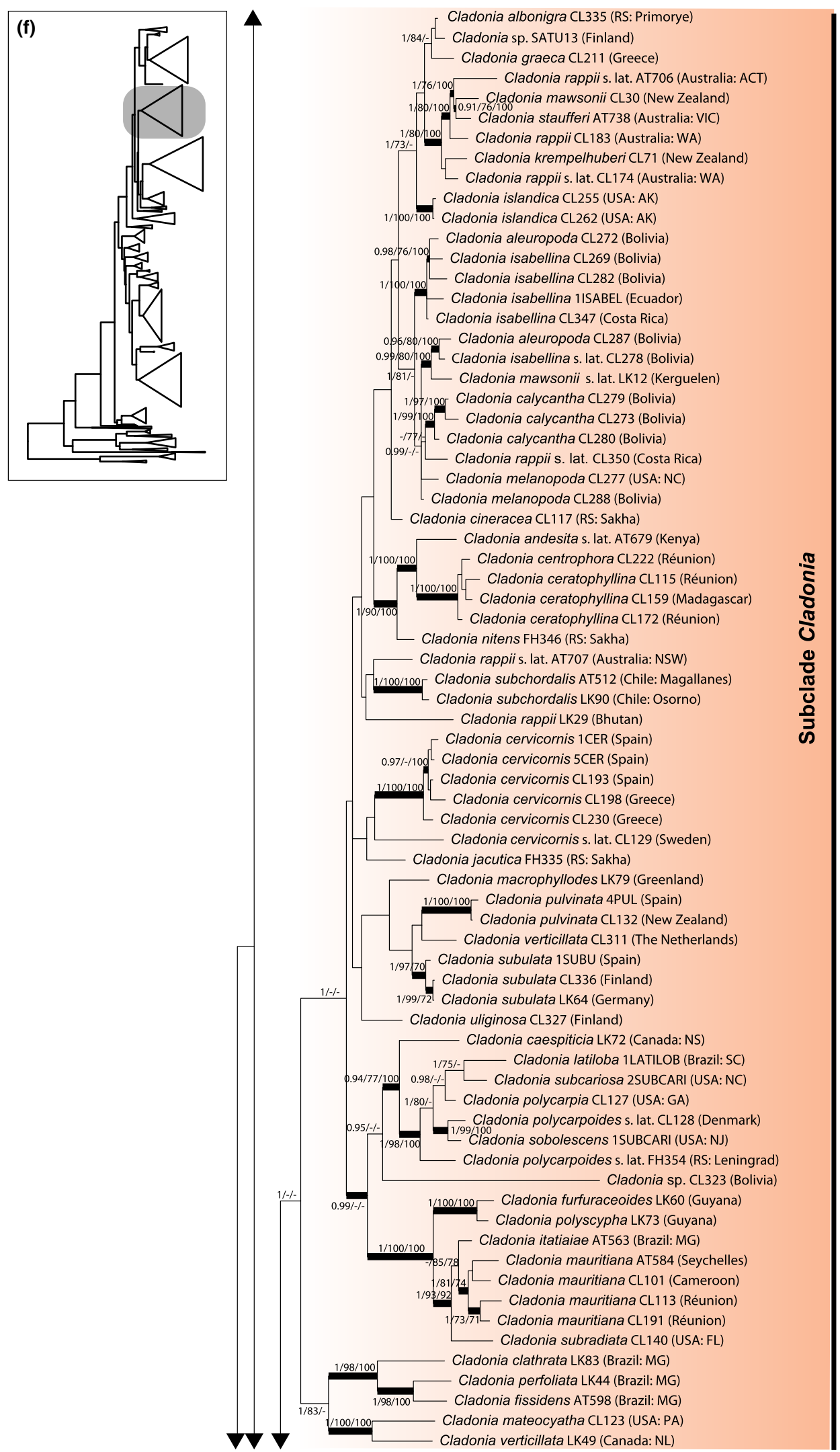

Fig. 2. Continued 

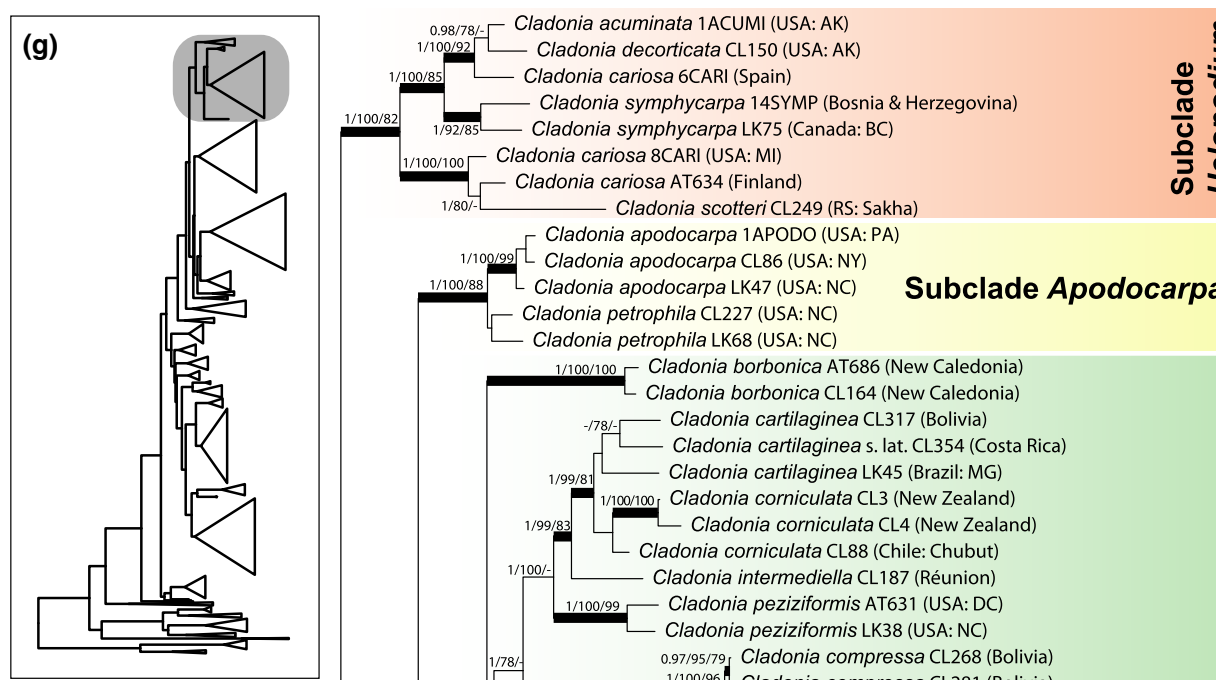

Subclade Apodocarpae

— Cladonia petrophila CL227 (USA: NC)
Cladonia petrophila LK68 (USA: NC)

1/100/100 - Cladonia borbonica AT686 (New Caledonia)

$\mathrm{C}$ Cladonia borbonica CL164 (New Caledonia)
Cladonia cartilaginea CL317 (Bolivia)

Cladonia cartilaginea CL317 (Bolivia)
Cladonia cartilaginea s. lat. CL354 (Costa Rica)

- Cladonia cartilaginea LK45 (Brazil: MG)

1/100/100, Cladonia corniculata CL3 (New Zealand)

1/99/83 L Cladonia corniculata CL4 (New Zealand)

Cladonia corniculata CL88 (Chile: Chubut)
Cladonia intermediella CL187 (Réunion)

1/100/99_ Cladonia peziziformis AT631 (USA: DC)

Cladonia peziziformis LK38 (USA: NC)

1/78/ $0.97 / 95 / 79$ Cladonia compressa CL268 (Bolivia)

$1 / 100 / 96$
Cladonia compressa CL281 (Bolivia) Cladonia nana LK34 (Brazil: MG)

Cladonia farinophylla CL325 (Bolivia) Cladonia farinophylla CL324 (Bolivia) Cladonia solida CL318 (Bolivia)

1/87/84 Cladonia scabriuscula CL12 (New Zealand)

1/87/74 L Cladonia scabriuscula LK58 (China: Hunan)

1/96/- L Cladonia scabriuscula LK11 (Canada: NL)

- Cladonia furcata 1FURC (Spain)

777. Cladonia stereoclada CL138 (Canary Is.)

1/100/79l Cladonia stereoclada 3STEREO (Canary Is.)

1/100/100 Cladonia farinacea 4FARIN (Chile: Magallanes)

Cladonia scabriuscula LK88 (Chile: Magallanes)

1/--1 Cladonia farinacea LK19 (Canada: NS)

98, 1/70/2990 donia multiformis LK70 (Canada: NS)

- Cladonia multiformis 1MULTIF (Canada: NS)

099/777 - Cladonia furcata AT638 (USA: GA)

1/75/100 1/-/- 1/99/100 Cladonia sp. 1HAMMER (Spain)

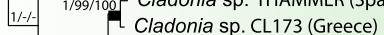

Cladonia humilis 7HUMIL (Portugal)

1/93/100 Cladonia humilis 2LAEVI (Spain)

10/100 Cladonia humilis 2LAEVI (Spain)

Cladonia humilis s. lat. 1PULVI (Spain)

$097 / 60 / 100-$ Cladonia humilis s. lat. 2POCI (Spain)

- Cladonia humilis s. lat. CL175 (Greece)

Cladonia furcata CL197 (Spain)

Cladonia corsicana CL110 (Canary Is.)

2/100 Cladonia corsicana COR1 (Spain)

Cladonia cyathomorpha 1CYATH (Spain)

Cladonia pulvinella 6NASHI (USA: CA)

1/100/100 Cladonia pulvinella CLCAL4 (USA: CA)

Lladonia pulvinella CLCAL1 (USA: CA)

$-1-100-$ Cladonia conista 1 HUMIL (Spain)

1/100/100 Cladonia conista FH398 (Lithuania)

Cladonia conista CL2 (New Zealand)

Cladonia conista LK56 (China: Hunan)

Cladonia dimorpha AT600 (Guyana)

- Cladonia subconistea 1KUROK (Japan: Kyushu)

- Cladonia subconistea LK48 (China: Hunan)

Cladonia subconistea 1SUBCONI (South Korea)

190/- Cladonia subconistea LK57 (China: Hunan)

1/100/100 Cladonia sp. CL25 (New Zealand)

Cladonia pyxidata s. lat. CL42 (New Zealand)

Cladonia pyxidata AT586 (Mexico: VC)

$$
\text { Cladonia sp. AT574 (Guyana) }
$$

$\underbrace{1 / 100 / 100}_{1 /-1-1}\left[\begin{array}{l}\text { Cladonia pseudofissa 1CORYMB (Thailand) } \\ \text { 1/100/100 Cladonia pseudofissa LK35 (Bhutan) }\end{array}\right.$

Cladonia mexicana CL321 (Bolivia)

Cladonia corymbescens AT680 (New Caledonia)

Cladonia corymbescens CL166 (New Caledonia)

- Cladonia ceratophylla CL188 (Réunion)

Cladonia ceratophylla LK37 (Brazil: MG)

Cladonia pityrophylla Cl 310 (Bolivi

- Cladonia pityrophylla LK40 (Brazil: MG) 
Table 3

Summary of support for the main clades in the different combined analyses of Cladoniaceae: posterior probability from Bayesian analysis/bootstrap from ML analysis/bootstrap from MP analysis/Jackknife from MP analysis in TNT

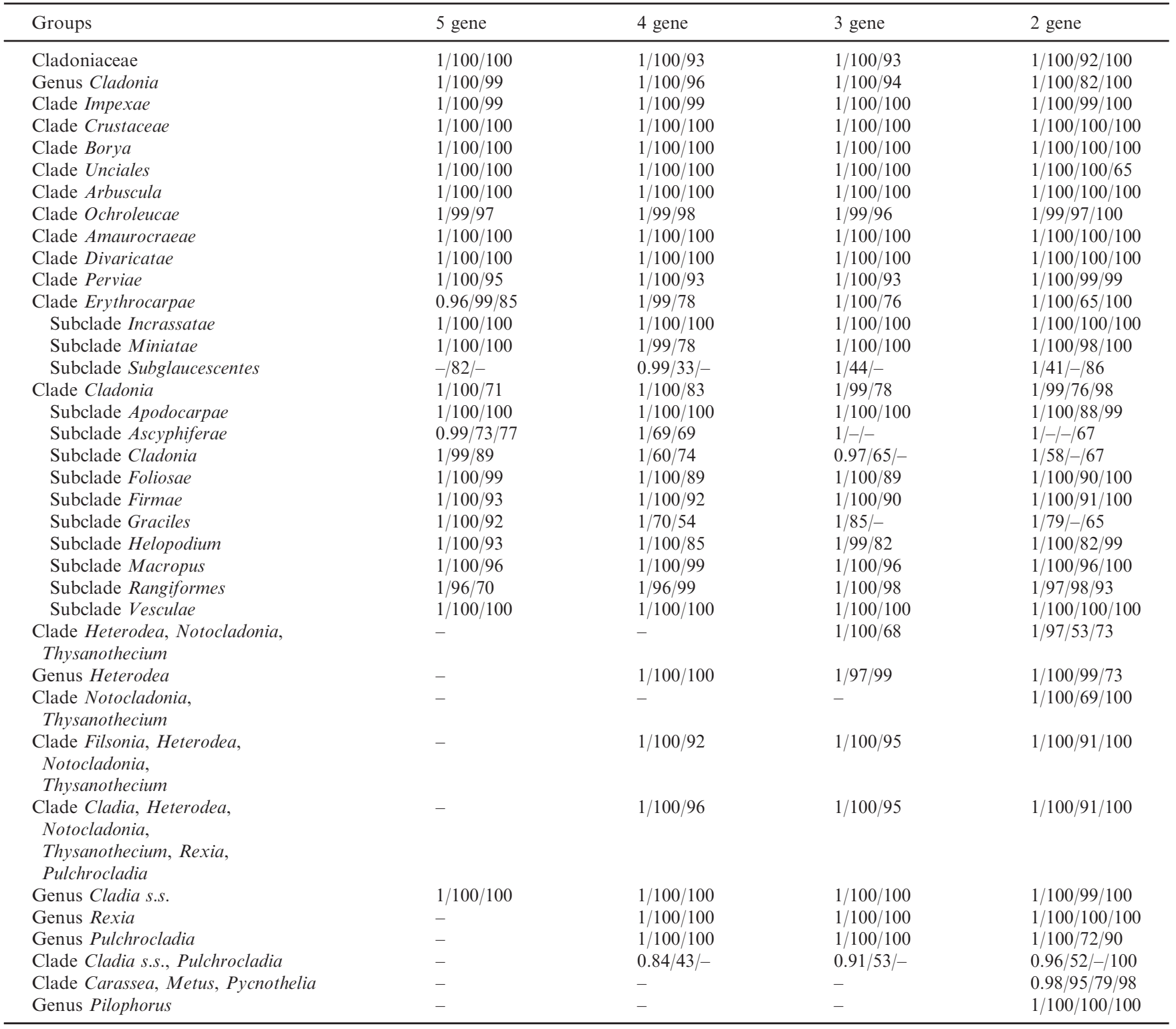

- Not applicable or bootstrap values $<50 \%$.

other Pilophorus species with a bootstrap support value of only $50 \%$. In the Bayesian analysis Pilophorus acicularis and $P$. cereolus formed a clade but the relationship of $P$. strumaticus was not resolved. The phylogenetic placement of Notocladonia was not resolved. Cladia beaugleholei and C. muelleri were only distantly related to the other species of Cladia. Cladia gorgonea, C. retipora, C. corallaizon, C. aggregata and C. sullivanii formed a clade with bootstrap values $<70 \%$ and $\mathrm{pp}<0.95$. The two species of Myelorrhiza were closely related and placed outside of Cladoniaceae, resolving as relatives to Bacidia.

\section{Discussion}

\section{Support values}

The phylogeny presented here is the most comprehensive for the Cladoniaceae to date with more species, specimens and molecular markers analysed. While not all species could be included, our sampling of Cladonia was more comprehensive than in the other genera, and the Clades were mostly supported. Therefore, we expect that most of the Clades named within Cladonia will remain stable in the future, even with more extensive 


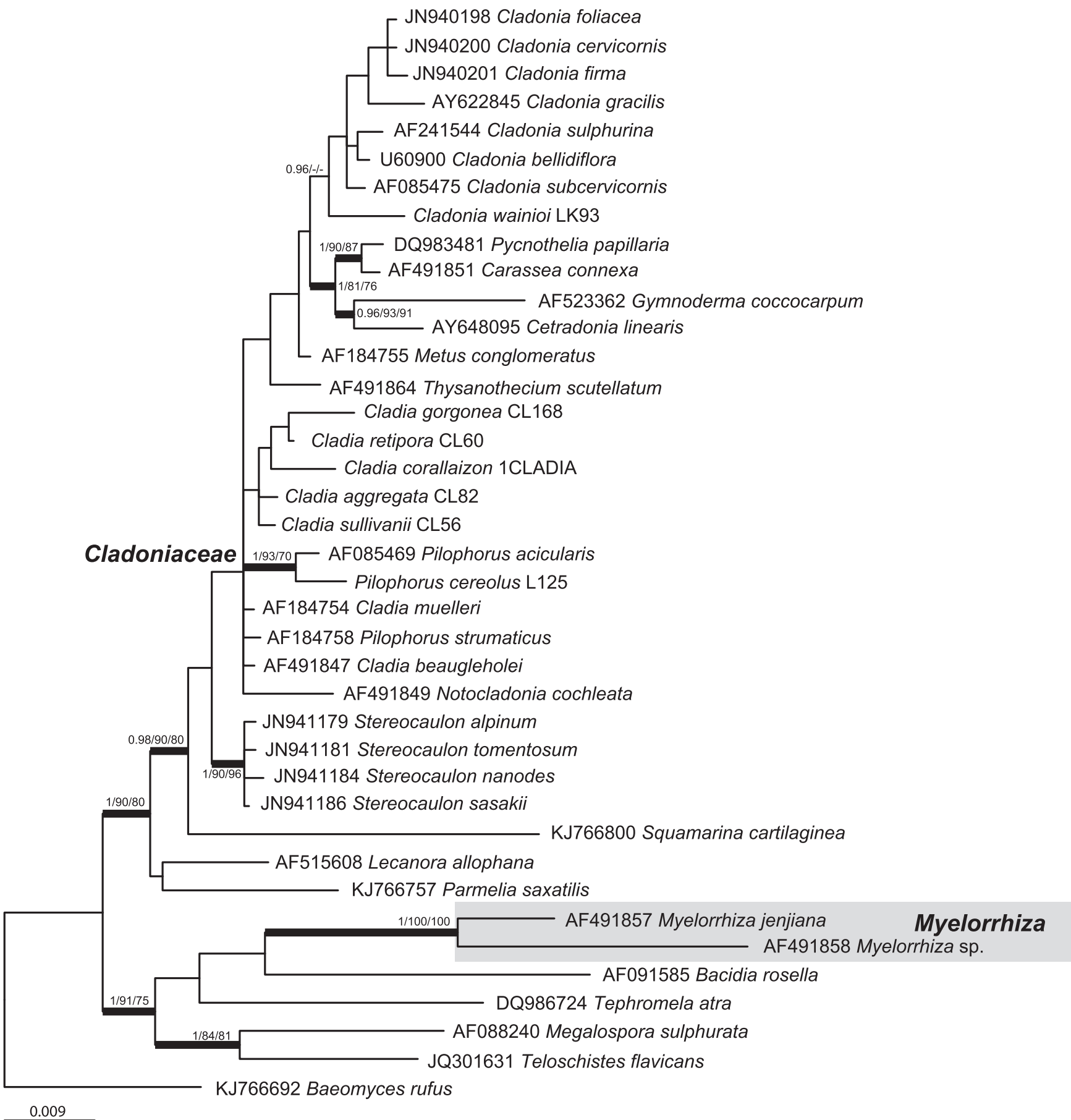

Fig. 3. Phylogeny of Cladoniaceae based on SSU rDNA. This is the $50 \%$ majority-rule consensus tree of Bayesian analysis. Branches supported with posterior probability $\geq 0.95$ and bootstrap $\geq 70 \%$ are indicated in bold.

sampling. The names of the Clades have been carefully chosen to follow the nomenclatural rules, so they could be used as sectional epithets within Cladonia, if desired. However, the position, or relationships, of the other genera of the Cladoniaceae might change substantially in the future if all genera can be analysed more comprehensively and the missing genera added.
The Clades delimited and discussed below are those with bootstrap and jackknife support values $\geq 70 \%$, and $\mathrm{pp} \geq 0.95$ (Table 3), with one exception, Clade Unciales (with $65 \%$ jackknife value in the $5+4+3+2$ gene dataset). Few Subclades (Ascyphiferae, Cladonia, Graciles and Subglaucescentes) lacked the threshold support values in any of the analyses (Table 3). The deep 
relationships among the clades had the greatest support in the 5 gene and $5+4$ gene analyses (Fig. 1), which had the most complete set of characters. The TNT $5+4+3+2+1$ gene analysis was less well resolved, possibly due to too many (almost 50) of terminals represented by only one marker.

In general, the support values from the MP analyses in PAUP were lower than the bootstrap values from ML and the pp from Bayesian analyses. This is probably due to using the "fast bootstrap" option in PAUP. It has been shown that "fast bootstrap" underestimates the support in comparison with the "heuristic" option (Mort et al., 2000; Freudenstein et al., 2004).

The significance of support values has been debated extensively (e.g. Carpenter, 1992; Hillis and Bull, 1993; Kluge and Wolf, 1993; Mort et al., 2000; Freudenstein et al., 2004). However, as discussed in detail by Grant and Kluge (2003), it is the inverse, the lack of support, which should be treated as the most valuable information obtained from these analyses. As Grant and Kluge emphasize, a lack of support represents part of a study's hypothesis that is ambiguous, as based on the currently available evidence, and deserves particular attention in the future.

\section{Circumscription of Cladoniaceae and relationships among the genera}

Our results based on five loci confirm the circumscription of Cladoniaceae as presented in previous studies (Stenroos and DePriest, 1998; Stenroos et al., 2002b; Guo and Kashiwadani, 2004; Zhou et al., 2006). The genera Carassea, Cladia, Cladonia, Metus, Notocladonia, Pilophorus, Pycnothelia and Thysanothecium were supported as belonging to Cladoniaceae. The early diverging clade Cladoniaceae is formed by taxa that have been referred to Cladia (including Heterodea and tentatively also Ramalinora), Notocladonia, Thysanothecium and Pilophorus (Fig. 2a). Pilophorus, represented by $P$. cereolus, was basal to this clade but its placement is unsettled. Its phylogenetic position in part of the analyses is congruent with previous phylogenies, in all of which it was related to Cladia (Stenroos and DePriest, 1998; Wedin et al., 2000; Stenroos et al., 2002b; Zhou et al., 2006; Parnmen et al., 2010; Miadlikowska et al., 2014). However, the relationship between these genera contrasts with their geographical distribution: while Cladia essentially is restricted to the Southern Hemisphere (Filson, 1981), Pilophorus only occurs in the Northern Hemisphere (Jahns, 1981).

In the SSU phylogeny, Pilophorus was represented by three species and was not monophyletic as P. strumaticus did not cluster with the other Pilophorus species. Pilophorus includes 12 species and it is the only genus of Cladoniaceae with cephalodia (Jahns, 1981). So far, the phylogenetic relationships of this genus are insufficiently known. Therefore, the monophyly of Pilophorus should be investigated with additional sampling of species and markers (R. Pino-Bodas, B. McCune, T. Ahti, in prep.).

The placement of Notocladonia in Cladoniaceae has long been doubted (Müller, 1896; Ahti, 1993, 2000), but Stenroos et al. (2002b) and our study support that this genus belongs to Cladoniaceae. Hammer (2003) suggested that Notocladonia is closely related to Thysanothecium. Notocladonia, with $N$. cochleata and $N$. undulata, has a bullate or squamulose primary thallus and unbranched or sparsely branched podetia. Both species contain usnic and divaricatic acids, and are confined to Australasia. Thysanothecium is confined to tropical areas in Asia and Australasia (Stenroos, 1988). Currently it includes four taxa, namely T. hookeri subsp. hookeri and subsp. xanthonicum, T. scutellatum and T. sorediatum. Hammer (2003) suggested, based on morphological characters, that Squamella is related to Thysanothecium and Notocladonia. However, the phylogenetic placement of the Australian endemic Squamella remains unknown.

Thysanothecium and Notocladonia diverge morphologically from Cladia s.s. Cladia lacks a primary thallus and has pseudopodetia with numerous small perforations (Filson, 1981), whereas Thysanothecium and Notocladonia have a dimorphic thallus, with a squamulose primary thallus, and podetia (Jahns, 1970a).

As found previously (Stenroos et al., 2002b; Miadlikowska et al., 2014), the sister clade to Cladonia consists of three small genera, namely Carassea, Pycnothelia and Metus. The latter two have morphological and chemical similarities: dimorphic thalli (expected but not observed in Carassea), with simple podetia or podetia branched near the apex, and containing atranorin and substances from the lichesterinic acid group (Galloway and James, 1987; Ahti, 2000). Carassea differs by having richly branched and highly anastomosing podetia, but still contains atranorin. The three genera have different geographical ranges. The monotypic Carassea is endemic to the mountains of southeast Brazil (Stenroos et al., 2002b), while Metus has an austral distribution with the Australasian M. conglomeratus and two Chilean species, M. efflorescens and M. pileatus. Pycnothelia is bipolar as a genus, with $P$. papillaria distributed in the Northern Hemisphere and $P$. caliginosa in Australasia. Sequence data of only one species of Metus, M. conglomeratus, and one of Pycnothelia, P. papillaria, were analysed, so we cannot confirm the monophyly of these genera.

As found by Zhou et al. (2006), Gymnoderma and Cetradonia were closely related in the SSU phylogeny. The relationship is supported by morphological characters associated with the asci, namely the shared nonamyloid axial body (Wei and Ahti, 2002; Peršoh et al., 2004). 
Myelorrhiza includes two Australian species, $M$. antrea and $M$. jenjiana. In the SSU analysis this genus was placed outside Cladoniaceae. Similar results were reported by Stenroos et al. (2002b). In their analysis Myelorrhiza was closely related to Bacidia. The phylogenetic position of Myelorrhiza was not supported as some morphological similarities with Phyllopsora (Ramalinaceae) were previously pointed out (Verdon and Elix, 1986). However, the type species $M$. antrea was not studied, and many authors continue to place this genus in Cladoniaceae (Lumbsch and Huhndorf, 2010; Kirk et al., 2013; Lücking et al., 2016; Wijayawardene et al., 2017, 2018). No new sequences of Myelorrhiza were generated in the present study, so new material will be necessary to clarify its position.

\section{Circumscription of Cladia}

Cladia was recently the target of several multilocus phylogenies (Lumbsch et al., 2010a; Parnmen et al., 2010, 2013). Parnmen et al. (2010) and Lumbsch et al. (2010a) confirmed that this genus and also Cladiaceae, recognized by Filson (1981), belong to Cladoniaceae. The authors also incorporated Heterodea and Ramalinora into Cladia. This was surprising, because Hererodea species are flat, foliose lichens, while Ramalinora is crustose, both very different from the typical fruticose Cladia. In fact, Filson (1981) placed Heterodea in a separate family Heterodeaceae, while Ramalinora was placed in various families (Ramalinaceae, Lumbsch et al., 1995; Lecanoraceae, Lumbsch and Huhndorf, 2007). However, there are several examples of lichens with different thallus growth forms that occur in the same family, such as Ramalinaceae, Roccellaceae and Stereocaulaceae (Myllys et al., 1999; Högnabba, 2006). In addition, Parnmen et al. (2010) and Lumbsch et al. (2010a) showed some morphological and anatomical similarities among Cladia, Heterodea and Ramalinora, supporting the close relationship of the three genera. It was proposed and accepted to conserve the generic name Cladia against the older name Heterodea to avoid confusion (Lumbsch et al., 2010b).

We have now discovered that Notocladonia and Thysanothecium fall within the Clade Cladia s.l., forming the sister group to Heterodea. Based on the tree topologies, we have several options for reconciling the taxonomy with the molecular phylogeny (see Fig. 2). One option is lumping the three genera into one. This treatment retains the taxonomic changes suggested by Parnmen and Lumbsch (2012). However, this option may be too drastic because Notocladonia and Thysanothecium are morphologically very disparate from Cladia. In addition, the type species of Thysanothecium (T. hookeri) was not available for analysis. Consequently, Thysanothecium is retained until the phylogenetic position of $T$. hookeri is studied. If instead Notocladonia and Thysanothecium are maintained as genera distinct from Cladia, the genus Heterodea also needs be maintained in order to reflect natural relationships. This option makes the nomenclatural proposal of Parnmen and Lumbsch (2012) superfluous. Based on the monophyly of the clades and the distinctive morphology of the two species of Heterodea, along with its close relationship with Notocladonia and Thysanothecium, we adopted the latter option for a revised classification of the clade. Consequently, the residual Cladia becomes paraphyletic and requires taxonomic revision.

Clade A of Cladia (Fig 2a), which is closely related to the clade formed by Heterodea, Notocladonia and Thysanothecium, includes two morphologically similar species, C. sullivanii and C. fuliginosa (Fig. 4). We suggest that this clade be considered as a genus separate from Cladia, and propose the name Rexia (described below). Clades B and C of Cladia (Fig. 2a) could be classified into one or two genera. The fact that the clade formed by $\mathrm{A}+\mathrm{B}$ is supported with bootstrap values $<70 \%$ and $\mathrm{pp}<0.85$ in most of the analyses (Table 3 ) proves that the stability of the clade is low, and some of the genera yet to be studied could be more closely related to clades $\mathrm{B}$ and $\mathrm{C}$ than they are to each other. Therefore, we keep clades B and C as separate genera in our taxonomic proposal. Clade B corresponds to Cladia s.s., while clade $\mathrm{C}$ corresponds to the new genus Pulchrocladia (described below). In this way, the genera that we recognize here constitute clades supported by bootstrap and jackknife values $\geq 70 \%$ and $\mathrm{pp} \geq 0.95$ (Table 3) and are also supported by either morphological or chemical characters, summarized in Table 4. Cladia s.s. is presently anticipated to include 19 species, among them the C. aggregata complex (Kantvilas and Elix, 1999; but see below). The species included in Cladia s.s. are characterized by hollow pseudopodetia, lack of an inner medulla, perforations that vary in size and abundance, and numerous chemotypes. All the species included in this genus show the unique development of the ascomata described in detail by Jahns (1970b): a second hymenium develops on the primary hymenium, a third develops on the second, and so on, in such a way that the apothecia are arranged in tiers. The species of Cladia s.s. were recently revised using molecular data (Parnmen et al., 2012, 2013) and all the recently described species belong to Cladia s.s. Nevertheless, not all morphological variation (Kantvilas and Elix, 1999) within this complex was included in the previous studies, and an increase in the number of Cladia s.s. species is anticipated.

\section{Overview of the phenotypic evolution of Cladonia}

The current circumscription of Cladonia is congruent with our results. Species of this genus are characterized 

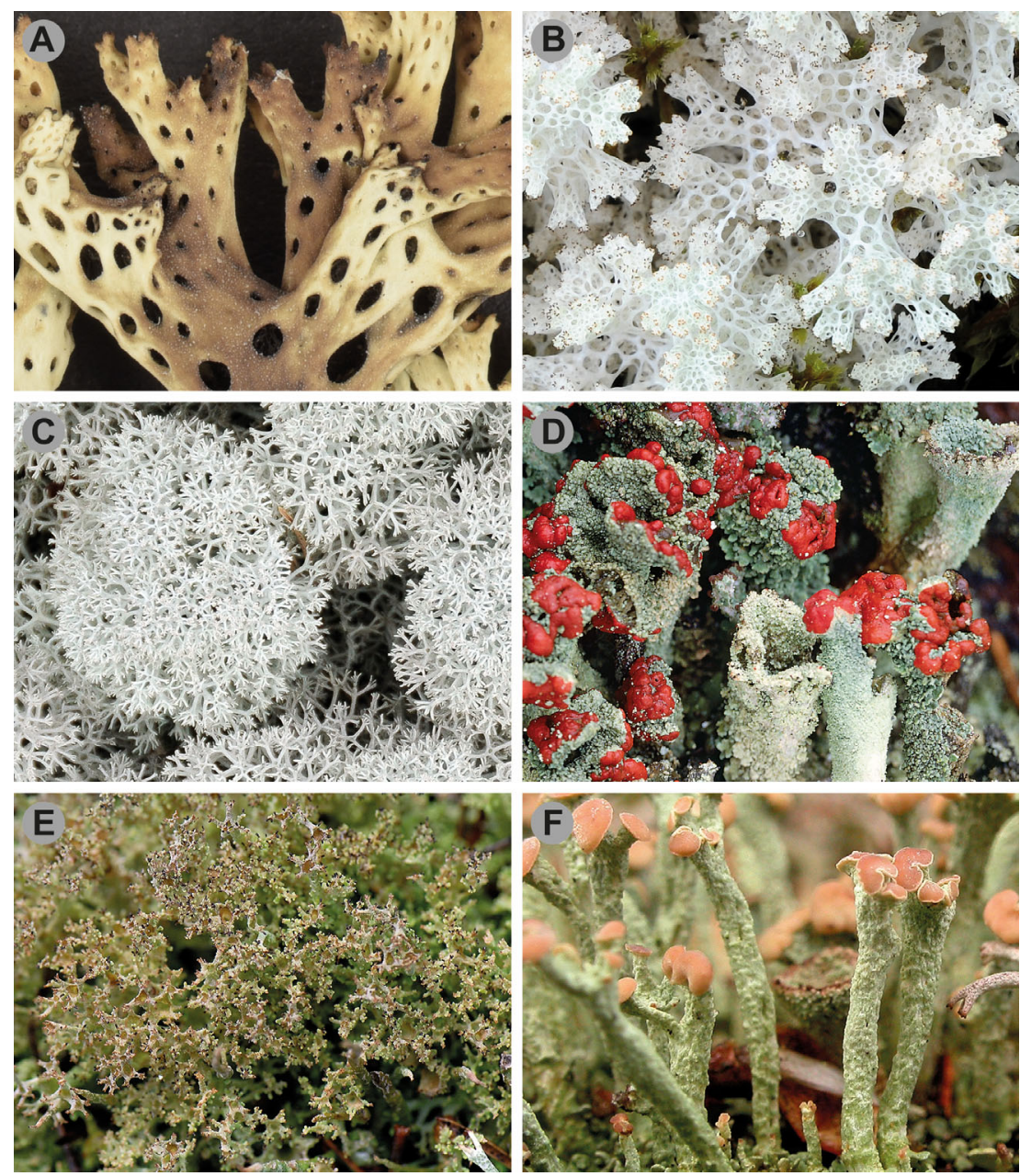

Fig. 4. Diversity of Cladoniaceae, illustrated by species of selected genera and Clades. A, Rexia sullivanii; B, Pulchrocladia retipora; C, Cladonia stellaris from Clade Impexae; D, Cladonia pleurota from Clade Erythrocarpae; E, Cladonia crispata var. crispata from Clade Perviae; F, Cladonia botrytes from Clade Ochroleucae. Photographs: A, C and E by S. Stenroos; B by J. Haapala; D by M. Korhonen; and F by E. Timdal.

by dimorphic thalli, with a squamulose primary thallus that is persistent or evanescent, and a secondary thallus formed by podetia that are notoriously variable in morphology (Figs. 4 and 5). The previous phylogenetic studies of the genus (Stenroos et al., 2002a, 2015; Guo and Kashiwadani, 2004; Beiggi and Piercey-Normore, 2007) showed that morphological similarities frequently do not reflect phylogenetic relationships. Therefore, it is important to examine a large number of species in order to understand the evolution of Cladonia. Our results support the same conclusion as previous studies, namely that the major clades contain enormous morphological and chemical variation. This could indicate that most of the species retain the genetic potential to vary into several different morphologies and to activate several pathways to synthesize numerous secondary metabolites. The fact that many morphologically and chemically variable species exist supports this hypothesis. Very few phenotypic characters are restricted to a unique clade, and instead they appeared several times independently during the evolution of the genus. Only in a few clades do species share synapomorphic characters: (1) Clade Ochroleucae, in which all species have ochraceous hymenial discs; (2) Clade Erythrocarpae, in which all species have red hymenial discs; and (3) Clade Borya, in which species have needle-like terpenoid crystals on the tips of podetia and a fibrous skeletal medullary tissue. Our results seem to indicate that brown apothecia are plesiomorphic, while red and ochraceous apothecia are apomorphic states. Both of the latter two states appeared only once during the evolution of Cladonia. However, our results do not support the long-held hypothesis that the ochraceous apothecia derived from the red ones by loss of rhodocladonic acid (Dahl, 1952; Ahti, 2000). Another character that restricts itself to a single clade is the presence of funnels, i.e. podetia with open axils and tips, often erroneously described as scyphi (cups). This character only appears in the Clade Perviae, although it is not shared by all 
species in the clade. Furthermore, the Cladina morphotype, characterized by densely branched, ecorticate podetia, has appeared at least three times independently. This type of podetium is shared by all species within the Clades in which it appears (Impexae, Arbuscula and Crustaceae).

Several classifications have been proposed to divide Cladonia into characterizable entities (see Vainio, 1897; Mattick, 1940; Ahti, 2000; Stenroos et al., 2002a). Our results do not support any of them as the characters historically used to define the sections within Cladonia (presence of scyphi, subulate podetia, branching patterns, dominant primary thallus, open or close axils, surface of podetia corticate, sorediate or covered with microsquamules, secondary metabolites, etc.) are not restricted to a single Clade of Cladonia.

\section{The largest genus of the family: Cladonia}

We distinguished 13 Clades in the genus Cladonia, some of which are divided into Subclades. We have clarified the nomenclature so that the most appropriate, existing names are applied, but at the same time we feel it is still too early to construct a formal subgeneric classification. Below, Clades of Cladonia are described, indicating their morphological and chemical variation, as well as the distribution patterns of the species included. These data were collected over decades of study both in the field and in countless herbaria around the world.

Clade Wainioa (Fig 2a) is sister to the remaining species in the genus and only includes Cladonia wainioi. This result is congruent with previous phylogenies (Subdivision I in Stenroos et al., 2002a; Athukorala et al., 2016). This species is distinct due to its cladinoid branching pattern in combination with the presence of merochlorophaeic acid. It is easily recognizable by these peculiar characters and therefore the risk of misidentification is probably low. It is distributed across western and north-eastern North America and in East Asia. Stenroos et al. (2002a) discussed the potential segregation of $C$. wainioi as a new genus, but ultimately they kept it in Cladonia and we follow their proposal.

Clade Delavaya (Fig. 2a) includes only one species and it is related to Clade Impexae. Similar results were obtained by Stenroos et al. (2002a) and Athukorala et al. (2016). Cladonia delavayi, the single species, is morphologically very distinct from the species of the Clade Impexae (Ahti, 1984). In fact, this species

Table 4

Diagnostic characters used to distinguish the new genera segregated from Cladia in the present paper: data are primarily based on the Cladia monograph by Filson (1981) and the treatment of Cladia in Galloway (1985), plus our own observations.

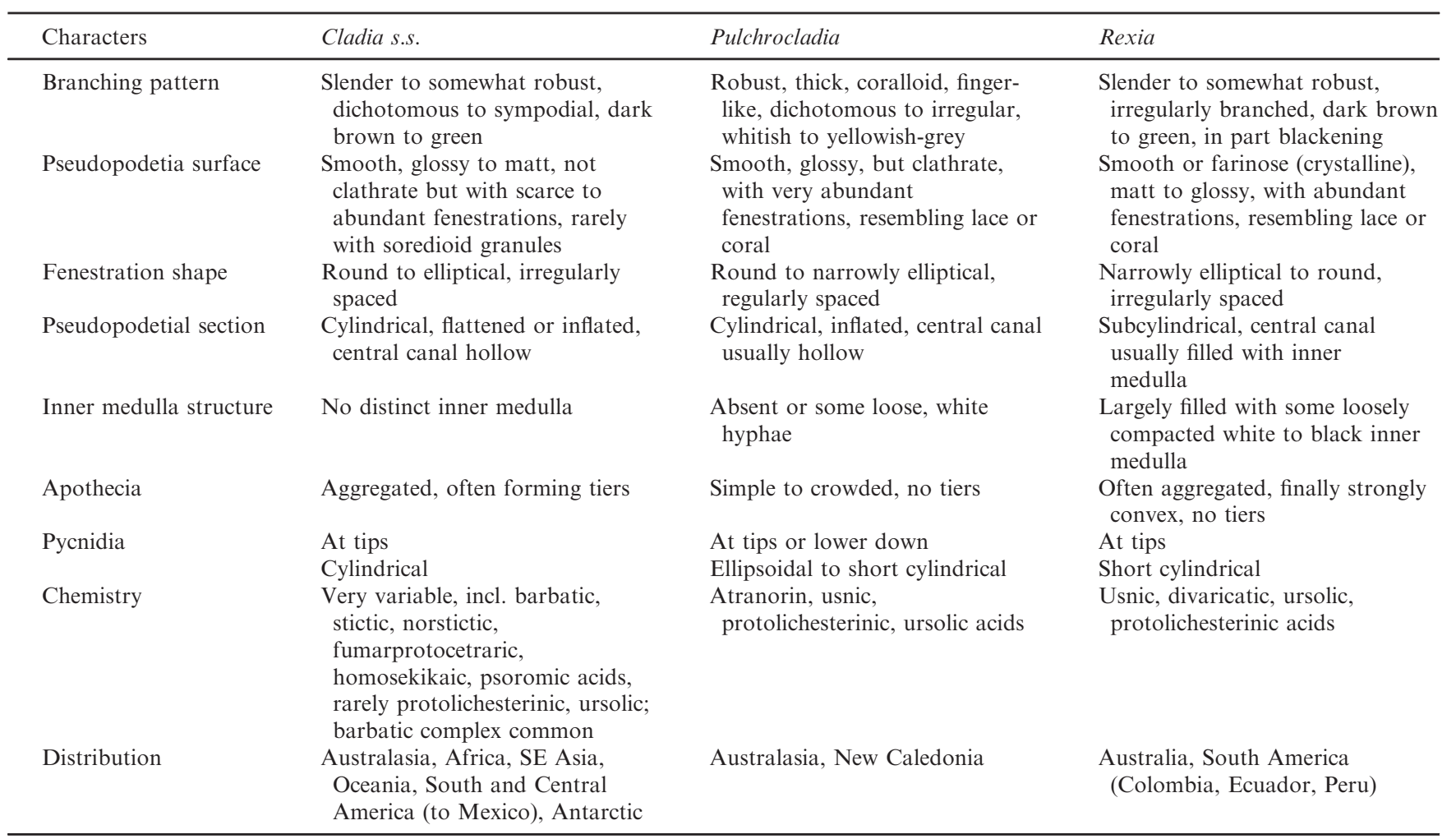



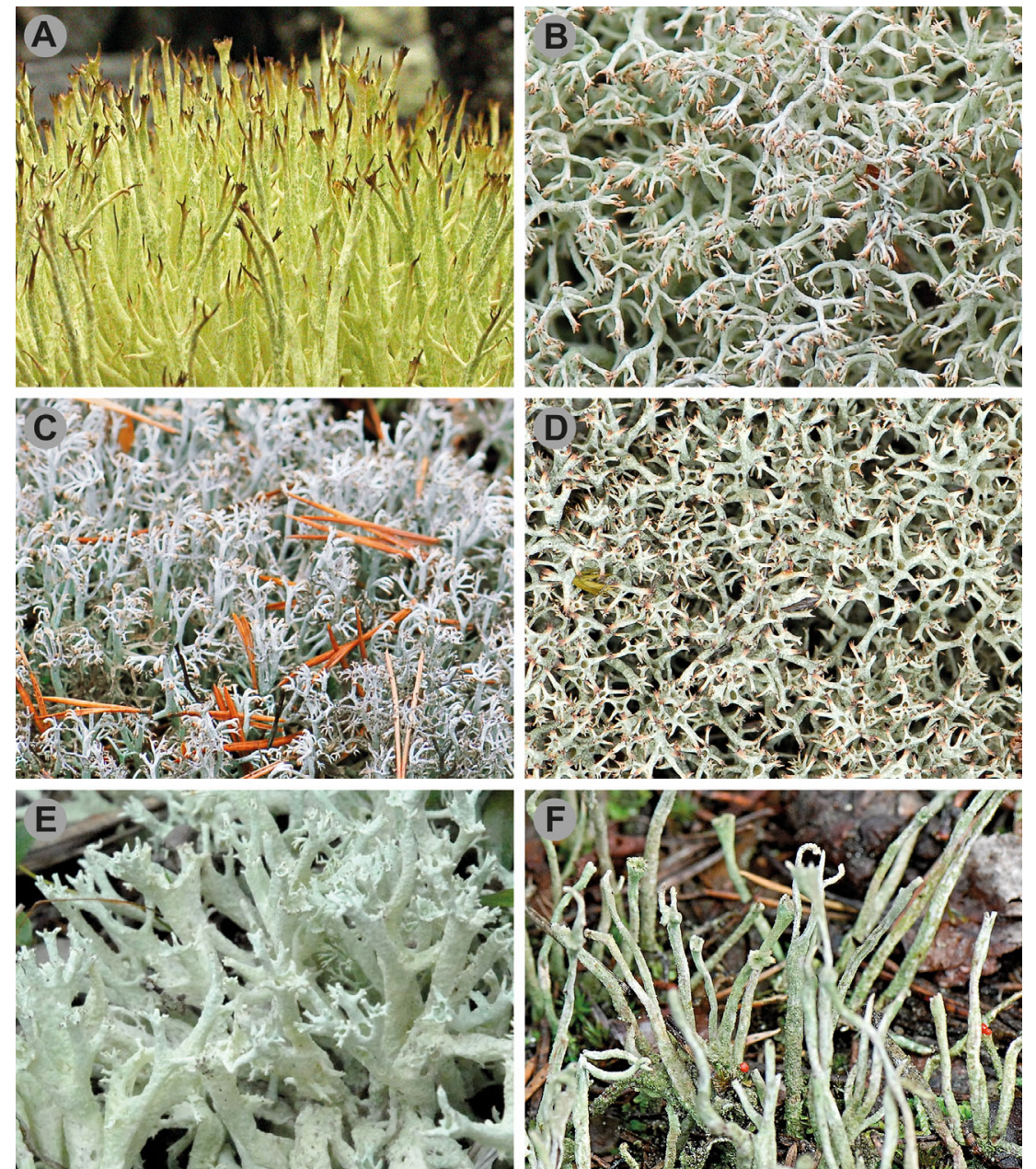

Fig. 5. Diversity of Cladonia, illustrated by species of selected Clades. A, C. amaurocraea from Clade Amaurocraeae; B, C. mitis from Clade Arbuscula; C, C. rangiferina from Clade Crustaceae; D, C. uncialis subsp. uncialis from Clade Unciales; E, C. boryi from Clade Borya; F, C. subulata from Clade Cladonia. Photographs: A by E. Timdal; B, D, E and F by S. Stenroos; and C by K. Jääskeläinen.

was earlier included in the section Unciales (des Abbayes, 1958). Consequently, Athukorala et al. (2016) suggested the creation of a new monotypic section for this Himalayan species, which is not phenotypically related to other described species. Its unique characters include the production of the depside 4-O-methylcryptochlorophaeic acid (Huovinen and Ahti, 1986b) as well as its unusual anatomy (depicted by des Abbayes, 1958).

Clade Impexae (Fig. 2a) includes species formerly of the genus Cladina, the so-called reindeer lichens. The non-monophyly of Cladina has been shown several times (Choisy, 1928; Hyvönen et al., 1995; DePriest et al., 2000; Stenroos et al., 2015; Athukorala et al., 2016). Our results confirm Cladina as polyphyletic with species spread over three separate clades, Clade Crustaceae, Clade Arbuscula and Clade Impexae. Impexae represents the old section Impexae sensu Ahti (2000). The members of Impexae are characterized by dichotomous or trichotomous branching patterns, and the presence of perlatolic and usnic acids (Ahti, 1984). Other phylogenetic studies have examined the relationships and species delimitation of some species included in this clade (Smith et al., 2012; Athukorala et al., 2016; Pino-Bodas et al., 2016). The species of this Clade have different geographical distributions: C. portentosa and $C$. stellaris are distributed in the Northern Hemisphere, mainly in boreal areas; $C$. mediterranea is restricted to South Europe, North Africa and Macaronesia; $C$. evansii is distributed in North and Central America; C. skottsbergii is confined to Hawaii; C. confusa has an austral distribution; $C$. terrae-novae is distributed in eastern North America, and C. pycnoclada is distributed in the southern part of South America and in the Antarctic region.

Clade Erythrocarpae (Fig. 2b) includes the major part of the traditional section Cocciferae (the correct name is Erythrocarpae at the section level). Species of 
this Clade are distinguished by red hymenial discs (colour caused by a naphthoquinone, rhodocladonic acid) and by the presence of dibenzofurans and various $\beta$ orcinol depsides such as thamnolic, barbatic and didymic acids, occasionally the terpene zeorin, and quite often usnic acid. Our analyses divide Erythrocarpae into three Subclades. The largest of them (Subglaucescentes) comprises most of the species. However, Subglaucescentes does not achieve acceptable support in all of our analyses. The two other Subclades contain fewer species, and are called here Miniatae and Incrassatae. Miniatae is a group of Neotropical species with corticate podetia. Many but not all of them have a red medulla and some grow directly on rock (Ahti, 2000). This group was thoroughly studied morphologically and chemically by Stenroos (1986a), and its monophyly was shown by Stenroos et al. (2002a). Subclade Incrassatae comprises just one species, $C$. incrassata, distinguished by its small, abundantly sorediate primary squamules, cylindrical and ascyphose podetia, and by containing usnic, didymic and squamatic acids (Ahti and Stenroos, 2013). It is found in Europe, East Asia and eastern part of North America.

The largest Subclade, Subglaucescentes, includes species with scyphose or ascyphose podetia with either corticate, sorediate or squamulose surfaces. This Subclade has a worldwide distribution (Ahti, 2000).

Clade Perviae (Fig. 2c) is essentially the same as the section Perviae, but many other, morphologically different species that have traditionally been included in other sections, such as the old Unciales, are now in Clade Perviae. This was also previously revealed in other phylogenies (Stenroos et al., 2002a, 2015). Our study is the first to confirm that several species traditionally included in the old section Perviae belong to this newly defined Clade (C. granulosa, C. subdelicatula, C. gigantea, C. poroscypha, C. hondoensis and C. varians). In general, Clade Perviae can be circumscribed by open podetial tips and axils, which are obvious in most species. In some species the openings are very wide and form so-called funnels that resemble scyphi (called "open scyphi" in many papers). Numerous species are squamulose or sorediate. Chemically, Perviae are fairly homogeneous (Huovinen and Ahti, 1982). Most species produce squamatic and/or thamnolic acid, both $\beta$-orcinol depsides, but barbatic acid also is common. However, a few peculiarities exist: didymic acid, which is common in Clade Erythrocarpae, is found in Cladonia metaminiata and C. piedadensis. The terpene zeorin, also common in Erythrocarpae, appears here in C. subfurcata. Finally, homosekikaic and sekikaic acids are only found in C. rigida, and usnic acid is randomly scattered across the Perviae. One clade including C. candelabrum, C. hedbergii, C. gigantea, C. medusina, C. pachyclados and Cladonia sp. represents an African element, widely represented in Madagascar and Réunion (Ahti, 1977; Stenroos, 1991). Other species in this clade have an Austral distribution (C. capitellata and C. subsubulata) but many are widespread (e.g. C. cenotea, C. squamosa and C. crispata).

Clade Divaricatae (Fig. 2c) was traditionally assigned to the section Unciales, but its distinct features were noted by Vainio as early as 1897 . These species appear peculiarly "spiny", the podetia are esquamose and esorediate, and usnic acid can be present or absent. Of the medullary substances, fumarprotocetraric acid is produced by C. albofuscescens, C. minarum, C. divaricata and C. ibitipocae, while the rest of the species produce thamnolic acid. Previous phylogenies showed that these species formed an evolutionary lineage (Stenroos et al., 2002a, 2015). However, a contradictory relationship inside the clade was apparent in previous phylogenies. In Stenroos et al. (2002a), C. consimilis was close to C. bahiana, while in our analyses, C. consimilis was closer to C. albofuscescens, C. minarum and $C$. divaricata, which are morphologically more similar species.

Clade Ochroleucae (Fig. 2d) is sister to the Clade Amaurocraeae, and this relationship has high support values in all concatenated analyses (Table 3 and Fig. 1). Ochroleucae includes seven species with pale ochraceous apothecia, a synapomorphic character in this clade. For a long time, these species were included in the section Cocciferae (Mattick, 1940; Dahl, 1952; Ahti, 2000), together with similar looking species with red apothecia. However, Vainio (1894) separated the ochraceous species into a different group. Our results show that Ochroleucae is only distantly related to the red-fruited Erythrocarpae, and therefore support Vainio's classification. Clades Amaurocraeae and Ochroleucae are chemically similar, with most of the species producing usnic and barbatic acids. Only C. pachyclados of the Ochroleucae produces grayanic acid, and C. elixii homosekikaic acid, while neither produce barbatic acid. Furthermore, C. carneola produces usnic acid and zeorin in addition to barbatic acid. The clade is morphologically fairly variable. For instance, C. carneola is always widely scyphose, whereas $C$. bacilliformis and $C$. elixii usually have simple podetia with acute tips; $C$. botrytes has simple or sparsely branched podetia always tipped with apothecia. Almost all species are sorediate or squamulose. Different geographical patterns are found: C. peltasta and C. pachyclados are known from East Africa, C. carneola and C. bacilliformis are bipolar, C. laii is restricted to Asia, C. elixii is endemic to New Zealand, and $C$. botrytes is widespread in the Northern Hemisphere.

Clade Amaurocraeae (Fig. 2d) is a peculiar clade with only one species, $C$. amaurocraea. Due to its morphology of richly branched podetia, an evanescent 
primary thallus, pale yellowish colour (usnic acid), smooth (esquamose, esorediate) cortex, and a tuft-like growth form, it was earlier considered a close relative of C. uncialis (Mattick, 1940). However, the two are only distantly related, as already shown by Stenroos et al. (2002a). The placement of C. amaurocraea has varied in different phylogenies. It formed its own clade related to Crustaceae (Stenroos et al., 2002a), and was closely related to $C$. peltastica (Stenroos et al., 2015) or to red-fruited species (Athukorala et al., 2016). Phenotypic characters suggested that $C$. amaurocraea could be phylogenetically related to C. nipponica (Stenroos et al., 2002a). However, the latter species is now included in Clade Borya. A close relationship with Unciales was anticipated, but $C$. amaurocraea was closer to Ochroleucae in all analyses. The unsampled C. hokkaidensis is morphologically similar to C. amaurocraea and it could be another member of the Clade Amaurocraeae. Cladonia amaurocraea can produce scyphi, has brown apothecia and red slime in its conidiomata, and contains usnic and barbatic acids. It has a circumpolar distribution, although a few records have been reported in tropical mountains of Asia (Litterski and Ahti, 2004).

Clade Arbuscula (Fig. 2d) consists of the widespread, arctic or boreal to temperate C. arbuscula, C. mitis and C. submitis, and the Neotropical C. densissima, C. kriegeri and C. arbuscula subsp. boliviana. Our results indicate that subsp. boliviana might not belong to C. arbuscula. However, as this subspecies is morphologically and chemically extremely variable (Ahti, 2000), more material is needed to clarify its taxonomic status. Although morphology suggests that C. arbuscula subsp. boliviana is different from $C$. incurva and C. arbuscula subsp. stictica, DNA sequences of these taxa must be compared. The phylogenetic relationships of C. arbuscula and C. mitis have been studied in detail by several authors (Myllys et al., 2003; Robertson and Piercey-Normore, 2007; Piercey-Normore et al., 2010), most revealing the lack of complete genetic divergence between the two at a species level, although in mixed colonies in the field they are commonly morphologically distinguishable.

Clade Crustaceae (Fig. 2d), the earliest epithet at the sectional level, corresponds to the former Cladina section Cladina and section Tenues (Ahti, 2000). Crustaceae includes species that are widely distributed in arctic and boreal to temperate areas (Cladonia ciliata, C. rangiferina and C. subtenuis), and many species have a Neotropical distribution (C. arcuata, C. argentea, C. rotundata, C. sprucei and C. rangiferina subsp. abbayesii). The circumscription of Crustaceae is similar to "clade D" found by Athukorala et al. (2016). These authors discussed the phenotypic variation of this clade in detail. The phylogenetic placement of C. sprucei and $C$. arcuata in Crustaceae is confirmed here.
Both taxa were included in the section Tenues by Ahti (2000). Kanz et al. (2015) found that the European specimens of $C$. rangiferina and C. stygia form different evolutionary lineages. However, most of the morphological variation of $C$. rangiferina is concentrated outside Europe (Ahti, 2000) and has not been examined yet.

Clade Unciales (Fig. 2d) was recently studied by Stenroos et al. (2015). According to their results, and confirmed by ours, this Clade appears to consist of only one species, C. uncialis, tentatively with two subspecies, subsp. uncialis and subsp. biuncialis. Cladonia uncialis contains usnic and squamatic acids or only usnic and rarely hypothamnolic acids, but the chemotypes do not fully correlate with the subspecies. The two subspecies basically differ by the type of branching, although this difference is not always clear. Cladonia uncialis occurs in the Northern Hemisphere, from arctic to temperate zones. The morphologically very similar C. steyermarkii was placed basal to C. uncialis (but with MP bootstrap values $<70 \%$ and jackknife $<70 \%$ in $5+4+3+2+1$ gene analyses) and it could not reliably be included in the Unciales. In earlier studies C. steyermarkii appeared in the Clade Divaricatae (Stenroos et al., 2002a, 2015). This Neotropical species usually contains usnic and thamnolic acids, and rarely additional squamatic and barbatic acids. Another species of the former section Unciales not studied here and which could be phylogenetically closely related to $C$. uncialis is C. papuana (Stenroos, 1986b).

Clade Borya (Fig. 2d) typically includes species that are fairly branched, lack soredia and podetial squamules, and commonly produce $\beta$-orcinol depsides, such as barbatic, squamatic or thamnolic acids; usnic acid (rarely absent). Most of the members of this clade are fairly versatile chemically, and chemotypes with stictic, fumarprotocetraric, squamatic and psoromic acids as major compounds are common. However, the feature that delimits this group is the production of terpenoid crystals at the podetial tips, a constant character present in all the species except C. solitaria. Clade Borya corresponds to the group earlier recognized as the C. boryi group (Ahti, 1973, 2000). The status of this clade was fully redefined by Stenroos et al. (2002a, 2015). Most of the species can be found in the Northern Hemisphere, but a small group of species (C. subreticulata, C. substellata, C. stenroosiae) grow in South America.

Clade Cladonia (Fig. 2d-g) contains the majority of Cladonia species. This clade includes species from the former sections Cladonia, Ascyphiferae and Helopodium, and some from the section Strepsiles sensu Ahti (2000). Podetial shapes and other podetial characteristics are extremely variable among and within species. Podetia can be present or absent, simple, sparsely or 
richly branched, scyphi present or absent, tips tapered, blunt, surface corticate, smooth, rough, sorediate, etc. There is no synapomorphy to diagnose the whole clade. We have named several Subclades within the Clade Cladonia, namely Helopodium, Apodocarpa, Ascyphiferae, Callosae, Cladonia, Graciles, Foliaceae, Vesculae, Firmae, Rangiformes and Macropus.

Subclade Helopodium (Fig. 2g) consists of species that can be considered the core species of the earlier defined section Helopodium, i.e. C. acuminata, C. cariosa, $C$. symphycarpa and $C$. decorticata, and also a species recently described and very similar to the others listed above, C. scotteri (Hansen and Ahti, 2011). They are characterized by fissured/striate podetia and a persistent primary thallus but these characters are not autapomorphic. Numerous chemotypes can be found among the species (Huovinen et al., 1989a; Osyczka and Skubała, 2011); atranorin, norstictic and psoromic acids are common. In addition, $C$. decorticata produces perlatolic acid. A molecular study was carried out on the $C$. cariosa complex, showing that more species than those currently described exist (Pino-Bodas et al., 2012a). Future molecular studies with an extended set of specimens are necessary.

Subclade Apodocarpa (Fig. 2g) only contains C. apodocarpa and C. petrophila, which were previously assigned to the section Helopodium due to their poorly developed podetia. Both contain fumarprotocetraric acid while atranorin is either present or absent; additionally, C. petrophila contains sphaerophorin, an uncommon substance in the genus Cladonia. In the earlier analyses this clade appeared in a similar position (Stenroos et al., 2002a). The independence of C. petrophila and C. apodocarpa has been examined using ITS rDNA (Lendemer and Hodkinson, 2009). However, these data did not have sufficient resolution to determine whether the two species form different lineages. Both species are distributed in temperate North America.

The name of Subclade Ascyphiferae (Fig. 2g) means "scyphi absent", and originally was used for sparsely or moderately branched species, such as C. furcata (type species of the old section). However, this Subclade includes many species that have well-developed scyphi. The species of the old section Ascyphiferae were shown to be non-monophyletic, as also seen in Stenroos et al. (2002a). The group now incorporates a variety of species with different morphologies and a number of different chemotypes. The major compounds are fumarprotocetraric, norstictic, psoromic, stictic, homosekikaic and merochlorophaeic acids. In addition, aliphatic acids, e.g. bourgeanic acid, can sometimes be found. One characteristic shared by many species is that fertile podetia have split and flattened areas, regardless of the general morphology of the podetia (i.e. C. corsicana, C. dimorpha, C. furcata,
C. humilis, C. pityrophylla). The species with wide scyphi (C. conista, C. humilis, C. pulvinella, C. subconistea) or species with a dominant primary thallus (C. ceratophylla, C. corsicana, C. pityrophylla) did not form monophyletic groups. Overall, the relationships inside the Subclade are not well resolved, so some changes in the topology of this clade are to be expected in the future. A supported clade in all the concatenated analyses is formed by a group of inconspicuous species, with a tropical distribution (C. cartilaginea, C. compressa, C. farinophylla, C. nana, C. peziziformis and C. solida). They have a persistent primary thallus and thick, sparsely branched, small podetia that have a corticate or areolate-corticate surface, and the occasional presence of phyllopodia. However, this clade also includes a species that breaks the pattern, $C$. corniculata, widespread in the Southern Hemisphere and with large, sorediate podetia (Ahti, 2000).

Subclade Callosae (Fig. 2g) only includes C. callosa. It is placed at the base of Subclades Apodocarpa and Ascyphiferae. It was previously included in the section Helopodium due to the (inconspicuously) fissured podetia that are unbranched or slightly branched. The presence of the fairly rare grayanic acid in this solitary species is noteworthy, as is its exclusively European distribution. It is not expected that any of the unsampled species will be placed to this clade.

Subclade Cladonia (Fig 2f) includes the type species of the genus, C. subulata. This clade includes species from the former sections Cladonia, Helopodium and Unciales (Ahti, 2000). All species with centrally proliferating scyphi, the so-called Cladonia verticillata group (Ahti, 2007), belong to this Subclade. On the other hand, species with the "classic" simple cup-shaped tip are absent. Similarly to the previous phylogeny (Stenroos et al., 2002a), C. subulata was found to be related to species with central proliferations; this is one of the few sorediate species in the Subclade. Most of the species in this Subclade produce substances of the fumarprotocetraric acid complex, and the cortical compound atranorin is sometimes also present. A small clade with C. subcariosa, C. polycarpoides and their allies is worth mentioning (bootstrap $\geq 70 \%$, pp $\geq 0.95$, jackknife $\geq 70 \%$ ). Previously included in the section Helopodium, they have a well-developed primary thallus, and are characterized by the production of compounds such as stictic, norstictic, connorstictic, psoromic and rangiformic acids. Cladonia subchordalis is unique in the Subclade by producing the cortical substance usnic acid. Consequently, it was previously assigned to the section Unciales. Another clade includes C. furfuraceoides, C. itatiaiae, C. mauritiana, C. polyscypha and C. subradiata (bootstrap $\geq 70 \%$, $\mathrm{pp} \geq 0.95$, jackknife $\geq 70 \%$ ). These species are characterized by slender and poorly branched podetia with a 
rough surface, covered with phyllidia, microsquamules, soredia or isidioid granules (Ahti, 2000).

Subclade Graciles (Fig. 2e) includes the C. gracilis group (Ahti, 1980), all with tall and fairly slender podetia, the C. pyxidata and C. chlorophaea groups, and other species with wide and well-formed scyphi. Species with scarcely branched podetia with a granulose or sorediate surface (such as C. fragosa or C. rei) are also present, as is a clade formed by $C$. dactylota and $C$. fruticulosa, characterized by generally scyphose and corticated podetia with tuberculose soralia (Ahti, 2000). Both sorediate and fully corticate species are present across the Subclade, and some species are coarsely granulose or plated.

Subclade Graciles is also chemically variable. Fumarprotocetraric acid is most commonly produced, but atranorin, homosekikaic, physodalic, psoromic, cryptochlorophaeic, paludosic, grayanic, merochlorophaeic and quaesitic acids are also found. Nevertheless, the morphologically circumscribed provisional groups are not monophyletic. For example, Cladonia alinii, an oceanic and mostly northern species from tundra and open forests (Ahti, 1980), very similar to C. gracilis, is only distantly related to it. Furthermore, the wide sampling in the present study confirms that the core of the C. pyxidata group (C. magyarica, C. monomorpha, C. pocillum and C. pyxidata) is placed in this clade, but C. pyxidata appears rather scattered and obviously presents taxonomic problems (Kotelko and PierceyNormore, 2010). The species of the Subclade Graciles have various distribution patterns. Numerous species are widespread, present on most of the continents, but occurring primarily in temperate to arctic regions (e.g. C. cornuta, C. gracilis, C. fimbriata, C. grayi, C. merochlorophaea, C. pocillum, C. pyxidata, C. rei), while others occur in tropical areas $(C$. dactylota, $C$. fruticulosa), and a few species are restricted to Australasia (C. darwinii, C. imbricata, C. tenerrima).

Some species of the Subclade Foliaceae (Fig. 2e) exhibit extremely large primary squamules that may comprise most of the thallus and sometimes form large rosettes or cushions. However, this clade also includes species with dominant scyphose podetia that often produce fairly large podetial squamules (Cladonia phyllophora, C. ramulosa). Usnic acid is commonly present in several species, such as C. foliacea, C. piedmontensis, C. robbinsii and Cladonia sp. Usually, this compound is accompanied by other substances, such as fumarprotocetraric acid (C. foliacea) or barbatic and homosekikaic acids (C. robbinsii). Other species only contain substances of the fumarprotocetraric acid complex (C. ramulosa, C. phyllophora). The presence of chlorovinetorin (a xanthone) in C. krogiana is noteworthy, as this substance is very rare in Cladonia. In the previous phylogeny this clade was scarcely sampled, but C. foliacea appeared close to C. phyllophora but not to C. robbinsii (Stenroos et al., 2002a). Although most of the species are restricted to the Northern Hemisphere (C.foliacea, C.piedmontensis, C. robbinsii), a putative new species, Cladonia sp., is only known from New Zealand. Cladonia ramulosa is widespread, but only the specimens from Europe occurred within this clade, while the specimens identified as C. ramulosa from other continents appeared in the Subclade Graciles (Fig. 2e).

Subclade Vesculae (Fig. 2e) is one of the smallest Subclades, represented by only two morphologically different species, C. vescula and C. signata. Cladonia vescula is a tiny, slender, sparsely branched species, whereas $C$. signata is tall and densely branched (Ahti et al., 2016). However, both are ecorticate, can produce fumarprotocetraric and homosekikaic acids, and occur in the Neotropics (C. signata has also been reported from Java, but that record is doubtful).

Subclade Firmae (Fig. 2d) also includes only two species, C. firma and C. subcervicornis. These species are morphologically and chemically similar, both have a well-developed primary thallus and they contain fumarprotocetraric acid and atranorin. In addition, their distribution is similar, being frequent in the Mediterranean area (Burgaz and Ahti, 2009). Cladonia firma has also been found in California, but the identity of these specimens must be confirmed.

Subclade Rangiformes (Fig. 2d) includes seven species, some of them characterized by large primary squamules often somewhat deformed. The podetia can be richly branched, turgid, fissured or squamulose. Cladonia rangiformis itself is perhaps the most peculiar species, with evanescent primary squamules, and fairly well-formed, branched podetia. Almost all the species produce atranorin as their major substance (absent from C. marionii). Some species additionally contain other compounds, such as fumarprotocetraric, bourgeanic, norstictic, stictic, protolichesterinic and psoromic acids, and zeorin. The distributions of these species are very disparate. While C. rangiformis and C. subturgida are present in West European-Mediterranean areas (Litterski and Ahti, 2004; Pino-Bodas et al., 2012b), C. turgida is a circumboreal species (Litterski and Ahti, 2004), C. neozelandica and C. sulcata are widespread in New Zealand and Tasmania (Galloway, 2007), and C. marionii occurs in subantarctic and the Antarctic region (Stenroos, 1993).

The Subclade Macropus (Fig. 2d) includes four species that lack any shared morphological features, although the two clades it contains (bootstrap values $\geq 70 \%$, pp $\geq 0.95$, jackknife $\geq 70 \%$ ) are phenetically well characterized. The name Macropus was introduced by Vainio (1894) for three species including C. macrophylla. Thomson (1968) added C. brevis. The present analyses confirmed that these species are closely related. Both lack scyphi, their podetia are 
somewhat stranded, they produce psoromic acid, and they are distributed in the Holarctic. Ahti (2000) considered that $C$. brevis was a mere chemotype of $C$. subcariosa and synonymized $C$. brevis with $C$. subcariosa, but our results show that the $C$. subcariosa group is distant from C. brevis. Another clade is formed by Cladonia asahinae and C. nashii, which both produce regular, wide scyphi and contain fumarprotocetraric acid. Cladonia nashii also produces atranorin, while C. asahinae has several chemotypes with various aliphatic acids (Huovinen et al., 1990; Brodo and Ahti, 1996). Both species are poorly known and their distribution has an oceanic tendency. Cladonia asahinae is found on most of the continents while $C$. nashii currently is only known from western North America.

\section{Future work in the Cladoniaceae}

Our results shed new light on the relationships of Cladoniaceae. In spite of the additional data presented here, the phylogeny of Cladoniaceae remains only partly resolved, mainly because several genera and numerous poorly known or rare species could not be sampled and many regions remain poorly explored (e.g. Himalayas, tropical South America and Africa). Furthermore, we were unable to produce a complete set of markers for all the terminal taxa.

The phylogenetic placement of the genera Calathaspis, Heteromyces, Ramalea, Sphaerophoropsis and Squamella remains unknown. Heteromyces was included in Cladoniaceae by Jahns (1970a), but some characters suggested that this genus could be related to Physcidia (Ramalinaceae). Sphaerophoropsis, with cylindrical pseudopodetia and two-celled spores (Vainio, 1880; Reinke, 1895; Lamb, 1954; Jahns, 1970a), has been related to various lichen genera (Lecidea, Toninia, Baeomyces, Mycoblastus), and Ahti (2000) excluded it from Cladoniaceae. Some authors also suggested that Ramalea does not belong to Cladoniaceae (Ahti, 2000; Hammer, 2003).

The difficulty of species delimitation is well known in the genus Cladonia. A number of studies were recently completed on several species complexes (Fontaine et al., 2010; Kotelko and Piercey-Normore, 2010; Piercey-Normore et al., 2010; Pino-Bodas et al., 2010, 2011, 2012a, 2013a, 2013b, 2015, 2016; Steinová et al., 2013; Stenroos et al., 2015). However, the molecular data did not always tell species apart consistently (Kotelko and Piercey-Normore, 2010; PinoBodas et al., 2012a, 2015). Our phylogenetic results underline not only the old, well-known delimitation problems, but have discovered new and unexpected ones. The present analyses found that Clade Cladonia contains most of the polyphyletic species and taxonomic problems. For instance, some specimens identified as of C.pyxidata appear in the Subclade
Ascyphiferae, while others appear in Subclade Graciles. It should be emphasized that there is great similarity between some specimens of $C$. pyxidata s.l. with some species of the C. humilis group (Pino-Bodas et al., 2013b). This is the reason why the specimens identified as C. pyxidata s.l., placed in the Subclade Ascyphiferae, could in fact belong to some species within the C. humilis group. Although quite a lot of work has been put into species groups, such as the Cladonia humilis group (Pino-Bodas et al., 2012b, 2013b), their complete variation has not yet been characterized. The Cladonia gracilis group was recently studied, with the conclusion that most of its species are polyphyletic (Kotelko et al., 2010; Pino-Bodas et al., 2011). The Cladonia verticillata group is still very poorly understood (Ahti, 2000). Many of the Neotropical species show high morphological or chemical variability (Ahti, 2000), and have not yet been studied in depth. Our results indicate that C. isabellina, C. aleuropoda and C. melanopoda are polyphyletic. As Ahti $(2000,2007)$ previously suggested, C. rappii also is polyphyletic. This species has a broad distribution (North America, South America, Asia, Asia, Africa and Melanesia) and the populations of the different regions could represent different taxa (Ahti, 2000). Cladonia ramulosa s.l. is one of the species with the greatest morphological variability (Ahti and Stenroos, 2013), characterized by scyphose podetia with abundant squamules on their surface. The European specimens of this species appear in the Subclade Foliosae, whereas the specimens from other continents group in the Subclade Graciles. Some of our specimens of C. ramulosa s.l. correspond to the C. pseudopityrea morphotype, so these taxa could be conspecific. The difficulty in identifying species of C. furcata and its assumed relatives C.farinacea, C. multiformis, C. scabriuscula and C. subrangiformis is well known. Pino-Bodas et al. (2015) proved that most species currently accepted in this group are polyphyletic and the high homoplasy of the taxonomic characters makes it difficult to delimit species.

In the Clade Perviae, the specimens of two morphologically divergent species, Cladonia capitellata and C. subsubulata, appeared surprisingly intermixed in our analyses. Cladonia capitellata is a species of the former section Unciales, it contains usnic acid, and has slender, subulate podetia. Cladonia subsubulata has whitish-brown, somewhat sturdy, simple to sparsely branched podetia, sometimes with narrow funnels, and contains thamnolic acid. Since genetic variation is low in this clade and the internal relationships are not supported, we amplified four additional markers $(\mathrm{mcm} 7$, $\beta$-tubulin, mtSSU, GDPH) for these two species. The results were similar to those with five loci: both species are polyphyletic (data not shown). Another unexpected polyphyletic species in the clade Perviae is C. cenotea, for which no taxonomic problems have previously 
been noted. The specimens of Cladonia crispata studied are not monophyletic. Although this species is seemingly easy to identify, several varieties and chemotypes have been described, and some South American specimens can be difficult to tell apart from other species (Brodo and Ahti, 1996) and other molecular studies have found similar problems (Bulat and Dudoreva, 1993; Stenroos et al., 2002a).

In the Clade Erythrocarpae, several species are polyphyletic. Steinová et al. (2013) found that C. deformis, $C$. pleurota, C. coccifera and C. diversa still need critical examination. Our results similarly show that the cosmopolitan C. coccifera is probably a complex of several undescribed species. Another problematic group includes Cladonia macilenta and C. floerkeana. Ahti (2000) already indicated that C. macilenta needs a worldwide revision. The polyphyly of $C$. didyma was expected as several authors indicated that it is morphologically very variable (Stenroos, 1986a; Ahti, 2000; Ahti and Stenroos, 2013).

In the Clade Arbuscula, Cladonia arbuscula and C. mitis have been treated several times (Myllys et al., 2003; Piercey-Normore et al., 2010; Smith et al., 2012; Athukorala et al., 2016) but there is still confusion regarding their taxonomic status. In addition, $C$. arbuscula has been divided into several subspecies (Ahti, 2000), which could indicate separate species, such as subsp. boliviana.

In the Clade Impexae, C. confusa was polyphyletic, as was also recently shown by Athukorala et al. (2016) and Pino-Bodas et al. (2016). This species, widely distributed and morphologically variable, has undergone several changes in its delimitation (Ahti, 1961, 1984, 2000). It led previous researchers to recognize up to seven different species (Ahti, 1961). Subsequently, Ahti (1984) synonymized all of them, but our results seem to indicate that the specimens from different geographical regions could represent different taxa (Fig. 2a). Additional sampling across their geographical ranges is required to assess this problem.

All the above-mentioned examples clearly indicate that identification and species delimitation in Cladonia is far from easy even for world experts. Once the large groups of phylogenetically related species have been established and appear stable, a detailed study of each, including numerous specimens per species, will be necessary. Only molecular studies that include all the phenotypic and geographical variation of the taxa will enable an accurate delimitation of Cladonia species, as well as an understanding of the evolution and distribution patterns of the genus. To include a number of specimens per species is unattainable in a family phylogeny like the present one. However, our results reveal numerous problems that highlight areas for future work. It is also likely that further problems will appear with additional phylogenetic studies. Special emphasis should be placed on the so-called cosmopolitan species, many of which will probably turn out to be taxonomic surprises. Finally, the multilocus sequence analyses of several Cladonia groups did not have enough resolution to establish the species' boundaries. We hypothesize that, due to the recent divergence of the species, they have a low genetic differentiation originating poor resolved phylogenies. Therefore, genome-level studies, sampling a large number of markers, will be of utmost importance to better understand the evolution of this notoriously variable and difficult lichen family.

\section{New taxa and nomenclatural novelties}

Rexia S. Stenroos, Pino-Bodas and Ahti, gen. nov. MycoBank No.: MB 828615

Type species. Rexia sullivanii (Müll. Arg.) S. Stenroos, Pino-Bodas and Ahti.

Diagnosis. Differs from Cladia by thick pseudopodetia with black to white, tightly packed, loosely woven medulla, commonly the production of usnic and divaricatic acids and a separate, wellsupported, monophyletic clade in a multilocus phylogeny.

Etymology. Refers to Rex B. Filson, an Australian lichenologist and the author of the monographs of Cladia and Heterodea.

Primary thallus papillate, soon evanescent. Pseudopodetia with indefinite growth (dying at base), 2.5$14 \mathrm{~cm}$ tall, 2-4 mm diameter, subcylindrical, angular, green to yellowish, easily becoming dark brown or black, rigid to horny (spongy when wet), somewhat branching, surface shiny or opaque, minutely crystalline, walls irregularly perforate. Medullary layer loosely woven, filling most of the hollow canal of the interior, black to white (near tips). Apothecia minute, terminal on upper branches of fertile pseudopodetia, lecideine, peltate to subspherical, brown to black, $0.25-0.5 \mathrm{~mm}$ diameter, ascospores simple, hyaline, 25$27 \times 5 \mu \mathrm{m}$. Conidiomata terminal on top branchlets of sterile pseudopodetia, conidia $6 \times 1 \mu \mathrm{m}$, curved or straight. Chemistry: Divaricatic acid, with usnic, ursolic, protolichesterinic and nordivaricatic acids as inconstant, are major secondary compounds. Distribution: South America, Australia and New Zealand.

Rexia sullivanii (Müll. Arg.) S. Stenroos, Pino-Bodas and Ahti, comb. nov.

MycoBank No.: MB 828616 
Cladonia sullivanii Müll. Arg., Flora 65: 294. 1 Jul 1882. - Cladia sullivanii (Müll. Arg.) W. Martin, Trans. \& Proc. Roy. Soc. New Zealand, Bot. 2: 44. 30 Nov 1962. - Type: Australia. Victoria, Grampian Mts., D. Sullivan 10 (G 00291123, holotype; UPS (?), US 00068156, isotypes).

\section{Figure $4 \mathrm{~A}$}

Primary thallus papillate, evanescent. Pseudopodetia up to $14 \mathrm{~cm}$ tall, greenish-yellow, straw-coloured, cinnamon-brown to black, rigid when dry, unevenly branched, forming clumps, wall moderately perforated, perforations irregularly round to ellipsoid; surface of pseudopodetia continuously corticate, opaque, with visible crystal deposits; inner medulla filling the pseudopodetium. Conidiomata terminal, with hyaline slime. Apothecia terminal with dark reddish-brown to brownish-black hymenia. Chemistry: Usnic, ursolic, protolichesterinic, nordivaricatic, and divaricatic acids all absent or present; additional unidentified triterpenoids may also be present. Distribution: Australasia: Australia (New South Wales, Tasmania, Victoria, Western Australia), New Zealand (North and South Islands). Also reported from southern South America (Filson, 1981), but probably incorrectly.

Rexia fuliginosa (Filson) S. Stenroos, Pino-Bodas and Ahti, comb. nov.

MycoBank No.: MB 828617

Cladia fuliginosa Filson, Victoria Naturalist 87: 325. 4 Nov 1970. - Type: Type: Australia. Tasmania, Cradle Mountain-Lake St. Clair National Park, Mt. Campbell, 1968 R. Filson 10854 (MEL 1001725, holotype; H 9500011, isotype).

Pseudopodetia up to $7 \mathrm{~cm}$ tall, green, becoming brown to black, partly with orange-brown tips, forming scattered clumps, rigid when dry, horny, irregularly branched, wall irregularly perforated, perforations broadly elliptical to round, irregularly spaced; surface of pseudopodetia discontinuously corticated, shiny, not crystalline; inner medulla filling most of the pseudopodetium. Conidiomata terminal, with hyaline slime. Hymenia reddish-brown to dark brown. Chemistry: Divaricatic and nordivaricatic acids, additional usnic acid in Neotropical populations. Distribution: Australasia: Australia (Australian Capital Territory, New South Wales, Tasmania, Victoria), New Zealand (Campbell Island, South Island). South America (Colombia, Ecuador, Peru).

Pulchrocladia S. Stenroos, Pino-Bodas, Lumbsch \&

Ahti, gen. nov.

MycoBank No.: MB 828618

Type species. Pulchrocladia retipora (Labill.) S. Stenroos, Pino-Bodas \& Ahti.
Diagnosis. Differs from Cladia by yellow, thick, tall pseudopodetia, which are richly fenestrate and reticulate and produce usnic acid and atranorin, and from Rexia by stranded, reticulate inner medulla, and the absence of divaricatic acid and presence of atranorin.

Etymology. Refers to the beautiful morphology of its species.

Primary thallus nodular, white, evanescent. Pseudopodetia (sterile and fertile similar) with indefinite growth (dying at base), 1-10 (15) cm tall, robust, coralloid, obtuse, branching system anastomosing, forming dense, extensive cushions, spongy when wet, yellow, grey, pale brown or white, walls clathrate from base to apex, perforations 5-11 per centimetre. Medulla thin, white, arachnoid, loosely filling central canal. Apothecia sparse, minute, crowded at apical branchlets, black, peltate, ascospores 25-27 × $5 \mu \mathrm{m}$, simple, hyaline, conidiomata terminal on small apical branchlets of sterile pseudopodetia, black, conidia $6 \times 1 \mu \mathrm{m}$, curved or straight, slime hyaline. Chemistry: Usnic acid, atranorin, protolichesterinic acid and ursolic acid as major secondary compounds. Distribution: Australasia and New Caledonia.

Pulchrocladia corallaizon (Filson) S. Stenroos, Pino-

Bodas \& Ahti, comb. nov.

MycoBank No.: MB 828619

Cladia corallaizon Filson, Victorian Naturalist 87: 324. 4 Nov. 1970. - Type: Australia. New South Wales, Narrandera to West Wyalong road, $45 \mathrm{~km} \mathrm{~N}$ of Ardlethan, 1963 R. Filson 5466 (MEL 25246, holotype).

Pseudopodetia up to $5 \mathrm{~cm}$ tall, pale grey to greenish-grey, becoming straw-coloured to pale brown, rigid when dry, horny, branched by dichotomy, forming pulvinate clumps or scattered clusters, wall richly perforated, perforations narrow, elliptical, regularly spaced; surface of pseudopodetia continuously corticate, soredia absent; inner medulla compacted, loosely filling pseudopodetium. Conidiomata terminal, with hyaline slime. Hymenia reddish-brown to black, infrequent. Chemistry: Usnic, protolichesterinic and ursolic acids, and atranorin. Distribution: Australasia: Australia (South Australia, Western Australia).

Pulchrocladia ferdinandii (Müll. Arg.) S. Stenroos, Pino-Bodas \& Ahti, comb. nov.

MycoBank No.: MB 828620

Cladonia ferdinandii Müll. Arg., Flora 65: 293. 1 Jul. 1882. - Cladia ferdinandii (Müll. Arg.) Filson, Victorian Naturalist 87: 325. 4 Nov. 1970. - Type: Australia. Western Australia, near Esperance Bay, 1864- 
1890 A. Dempster s.n. (G 00291121, holotype; MEL 1015495, isotype).

\section{Figure $4 B$}

Pseudopodetia up to $15 \mathrm{~cm}$ tall, creamy white, yellowing at tips, rigid when dry, horny, irregularly branched, forming pulvinate clumps, wall richly perforated, almost reticulate, perforations large, irregularly rounded; surface of pseudopodetia continuously corticate, soredia absent. Conidiomata terminal, with hyaline slime. Hymenia not known. Chemistry: Usnic and ursolic acids, and atranorin. Distribution: Australia: Australian Capital Territory, New South Wales, Queensland, South Australia, Western Australia.

Pulchrocladia retipora (Labill.) S. Stenroos, PinoBodas \& Ahti, comb. nov.

MycoBank No.: MB 828621

Baeomyces retiporus ("reteporus") Labill., Nov. Holl. P1. 2: 110. Feb. 1806. - Cladia retipora (Labill.) Nyl., Compt. Rend. Hebd. Séances Acad. Sci. 83: 88. Jul. 1876. - Type: Australia. Tasmania ("Cap van Diemen, nouv. Hollande"), 1792 J. J. H. Labillardière s.n. (PC, lectotype designated by Filson, 1981; BM, FH-Tuck, FI-W(2), G, PC, PC-Lenormand, PC-Montagne, isolectotypes; LINN-Sm 1710.17, possible isolectotype).

Pseudopodetia up to $5 \mathrm{~cm}$ tall, white to pale grey, sometimes tinged pinkish or yellowing or superficially blackening at tips, rigid when dry, horny, richly branched irregularly or by dichotomy, forming pulvinate clumps, wall richly perforated, almost reticulate, perforations large, round to ellipsoidal; surface of pseudopodetia continuously corticate, soredia absent; inner medulla stranded. Conidiomata terminal, with hyaline slime. Hymenia dark reddish-brown to black. Chemistry: Usnic, protolichesterinic and ursolic acids, and atranorin, often also additional rangiformic and norrangiformic acids. Distribution: Pacific: New Caledonia. Australasia: Australia (Australian Capital Territory, New South Wales, Queensland, Victoria, Tasmania), New Zealand (North and South Islands, Antipodes Island, Auckland Islands, Campbell Island, Chatham Islands).

\section{Acknowledgments}

We thank A. R. Burgaz, M. Christenhusz, P. Clerc, A. Flakus, M. Kukwa, F. Schumm and S. Talbot for providing specimens for this study and S. Parnmen for providing sequences. R. Strickman and D. Weckman are thanked for their assistance in the molecular laboratory. We also thank K. Jääskeläinen, E. Timdal, R. Haugan and J. Haapala for providing some of the photographs, L. Helynranta for preparing the photo plates and D. Haugland and T. Sydney for improving the language of the manuscript. M. P. Martín is thanked for hosting S. Stenroos during her stay in Madrid. This study received funding from the Academy of Finland Grant No. 211171 and the SYNTHESYS ES-TAF-922 (S. Stenroos); the European Union's Seventh Framework Program under Grant No. PIEFGA-2013-625653; Juan de la CiervaIncorporación no. 2015-23526; Spanish Ministry of Economy, Industry and Competitiveness (MINECO) (R. Pino-Bodas); and the project CGL2013-41839-P led by A. R. Burgaz (T. Ahti and R. Pino-Bodas). The agencies providing funding are greatly acknowledged.

\section{References}

Abbayes, H., 1958. Résultats des expéditions scientifiques genevoises au Népal en 1952 et 1954 (Partie botanique) 12. Cladonia (Lichen). Candollea 16, 201-209.

Ahti, T., 1961. Taxonomic studies on reindeer lichens (Cladonia, subgenus Cladina). Ann. Bot. Soc. Zool.-Bot. Fenn. Vanamo 32 (1), $1-160$.

Ahti, T., 1973. Taxonomic notes on some species of Cladonia, subsect. Unciales. Ann. Bot. Fenn. 10, 163-184.

Ahti, T., 1977. The Cladonia gorgonina group and C. gigantea in East Africa. Lichenologist 9, 1-15.

Ahti, T., 1980. Taxonomic revision of Cladonia gracilis and its allies. Ann. Bot. Fenn. 17, 195-243.

Ahti, T., 1984. The status of Cladina as a genus segregated from Cladonia. Beih. Nova Hedwigia 79, 25-61.

Ahti, T., 1993. Names in current use in the Cladoniaceae (lichenforming Ascomycetes) in the ranks of genus to variety. Regnum Veg. 128, 58-106.

Ahti, T., 2000. Cladoniaceae. Fl. Neotr. Monogr. 78, 1-362.

Ahti, T., 2007. Further studies on the Cladonia verticillata group (Lecanorales) in East Asia and western North America. Biblioth. Lichen. 96, 5-19.

Ahti, T., Stenroos, S., 2013. Cladoniaceae. In: Ahti, T., Stenroos, S., Moberg, R. (Eds), Nordic Lichen Flora, Vol. 5, Museum of Evolution, Uppsala University, Uppsala, pp. 1-117.

Ahti, T., Pino-Bodas, R., Flakus, A., Stenroos, S., 2016. Additions to the global diversity of Cladonia. Lichenologist 48, 517-526.

Alfaro, M.E., Zoller, S., Lutzoni, F., 2003. Bayes or bootstrap? A simulation study comparing the performance of Bayesian Markov chain Monte Carlo sampling and bootstrapping in assessing phylogenetic confidence. Mol. Biol. Evol. 20, 255-266.

Arup, U., Ekman, S., Grube, M., Mattsson, J.E., Wedin, M., 2007. The sister group relation of Parmeliaceae (Lecanorales, Ascomycota). Mycologia 99, 42-49.

Athukorala, S.N., Pino-Bodas, R., Stenroos, S., Ahti, T., PierceyNormore, M.D., 2016. Phylogenetic relationships among reindeer lichens of North America. Lichenologist 48, 209-227.

Begerow, D., John, B., Oberwinkler, F., 2004. Evolutionary relationships among $\beta$-tubulin gene sequences of basidiomycetous fungi. Mycol. Res. 108, 1257-1263.

Beiggi, S., Piercey-Normore, M., 2007. Evolution of ITS ribosomal RNA secondary structures in fungal and algal symbionts of selected species of Cladonia sect. Cladonia (Cladoniaceae, Ascomycotina). J. Mol. Evol. 64, 528-542.

Brodo, I.M., Ahti, T., 1996. Lichens and lichenicolous fungi of the Queen Charlotte Islands, British Columbia, Canada. 2. The Cladoniaceae. Can. J. Bot. 74, 1147-1180.

Bulat, S., Dudoreva, T., 1993. Cladonia cenotea in the Kolsky peninsula: differentiation by the UP PCR (RAPD PCR like) technique. Abstr. 12th Int. Conf. Mycol. Lichenol., Vilnius, p. 135. 
Burgaz, A.R., Ahti, T., 2009. Cladoniaceae. Fl. Lich. Ibérica, Vol. 4. Sociedad Española de Liquenología, Madrid.

Carpenter, J.M., 1992. Random cladistics. Cladistics 8, 147-153.

Choisy, M., 1928. Sur le phylétisme des Ascomycètes du genre Cladonia (Lichens). Bull. Soc. Mycol. France 43, 267-271.

Culberson, C.F., 1969. Chemical and Botanical Guide to Lichen Products. University of North Carolina Press, Chapel Hill.

Culberson, C.F., 1970. Supplement to Chemical and Botanical Guide to Lichen Products. Bryologist 73, 177-377.

Culberson, C.F., 1986. Biogenetic relationships of the lichen substances in the framework of systematics. Bryologist 89, 91-98.

Culberson, C.F., Culberson, W.L., Johnson, A., 1977. Second Supplement to Chemical and Botanical Guide to Lichen Products. American Bryological and Lichenological Society, Missouri Botanical Garden, St. Louis.

Dahl, E., 1952. On the use of lichen chemistry in lichen systematics. Rev. Bryol. Lichénol. 21, 119-134.

DePriest, P.T., 1993. Molecular innovations in lichen systematics: the use of ribosomal and intron nucleotide sequences in the Cladonia chlorophaea complex. Bryologist 96, 314-325.

DePriest, P.T., 1994. Variation in the Cladonia chlorophaea complex. II: Ribosomal DNA variation in a southern Appalachian population. Bryologist 97, 117-126.

DePriest, P.T., Piercey-Normore, M., Sikaroodi, M., Kärkkäinen, K., Oksanen, I., Yahr, R., Ahti, T. 2000. Phylogenetic relationships among sections of Cladonia and Cladina. In: Progress and Problems in Lichenology at the Turn of the Millenium: The Fourth IAL Symposium, Abstract Book. University of Barcelona, Spain.

Ekman, S., Andersen, H., Wedin, M., 2008. The limitations of ancestral state reconstruction and the evolution of the ascus in the Lecanorales (lichenized Ascomycota). Syst. Biol. 57, 141-156.

Farris, J.S., Albert, V.A., Källersjö, M., Lipscomb, D., Kluge, A.G., 1996. Parsimony jackknifing outperforms neighbor-joining. Cladistics 12, 99-124.

Filson, R.B., 1978. A revision of the genus Heterodea Nyl. Lichenologist 10, 13-25.

Filson, R.B., 1981. A revision of the lichen genus Cladia Nyl. J. Hattori Bot. Lab. 49, 1-75.

Fontaine, K.M., Ahti, T., Piercey-Normore, M.D., 2010. Convergent evolution in Cladonia gracilis and allies. Lichenologist 42, 323338.

Freudenstein, J.V., van den Berg, C., Goldman, D.H., Kores, P.J., Molvray, M., Chase, M., 2004. An expanded plastid DNA phylogeny of Orchidaceae and analysis of jackknife branch support strategy. Am. J. Bot. 91, 149-157.

Galloway, D.J., 1985. Flora of New Zealand Lichens. P.D. Hasselberg, Government Printer, Wellington.

Galloway, D.J., 2007. Flora of New Zealand Lichens. Revised second edition, Vol. 1. Manaaki Whenua Press, Lincoln, New Zealand.

Galloway, D.J., James, P.W., 1987. Metus, a new austral lichen genus and notes on an Australasian species of Pycnothelia. Notes Roy. Bot. Gard. Edinburgh 44, 561-579.

Gardes, M., Bruns, T.D., 1993. ITS primers with enhanced specificity for basidiomycetes - application to the identification of mycorrhizae and rusts. Molec. Ecol. 2, 113-118.

Gargas, A., DePriest, P.T., 1996. A nomenclature for fungal PCR primers with examples from intron-containing SSU rDNA. Mycologia 88, 745-748.

Goloboff, P.A., 1999. Analyzing large data sets in reasonable times: solutions for composite optima. Cladistics 15, 415-428.

Goloboff, P.A., Catalano, S.A., 2016. TNT version 1.5, including a full implementation of phylogenetic morphometrics. Cladistics 32, 221-238

Goloboff, P.A., Farris, J.S., Nixon, K., 2008. TNT, a free program for phylogenetic analysis. Cladistics 24, 774-786.

Grant, T., Kluge, A.G., 2003. Data exploration in phylogenetic inference: scientific, heuristic, or neither. Cladistics 19, 379-418.

Grube, M., Hawksworth, D.L., 2007. Trouble with lichen: the re-evaluation and re-interpretation of thallus form and fruit body types in the molecular era. Mycol. Res. 111, 11161132 .

Guo, S., Kashiwadani, H., 2004. Recent study on the phylogeny of the genus Cladonia (s.1.) with the emphasis on the integrative biology. Nat. Sci. Mus. Monogr., Tokyo 24, 207-225.

Hall, T.A., 1999. BioEdit: a user-friendly biological sequence alignment editor and analysis program for Windows 95/98/NT. Nucleic Acids Symp. Ser. 41, 95-98.

Hammer, S., 1993. Development in Cladonia ochrochlora. Mycologia $85,84-92$.

Hammer, S., 1995. Primary tissue and the structure of the podetium in Cladonia. Mycologia 87, 46-53.

Hammer, S., 2003. Notocladonia, a new genus in the Cladoniaceae. Bryologist 106, 162-167.

Hansen, E.S., Ahti, T., 2011. A contribution to the lichen genus Cladonia in Greenland and new records from other northern regions. Graphis Scripta 23, 56-64.

Henssen, A., Jahns, H.M., 1973. Lichenes. Eine Einführung in die Flechtenkunde. G. Thieme, Stuttgart.

Hillis, D.M., Bull, J.T., 1993. An empirical test of bootstrapping as a method for assessing confidence in phylogenetic analysis. Syst. Biol. 42, 182-192.

Hofstetter, V., Miadlikowska, J., Kauff, F., Lutzoni, F., 2007. Phylogenetic comparison of protein-coding versus ribosomal RNA-coding sequence data: a case study of the Lecanoromycetes (Ascomycota). Mol. Phylogenet. Evol. 44, 412-426.

Högnabba, F., 2006. Molecular phylogeny of the genus Stereocaulon (Stereocaulaceae, lichenized ascomycetes). Mycol. Res. 110, 1080-1092.

Huneck, S., Yoshimura, I., 1996. Identification of lichen substances. In Huneck, S., Yoshimura, I. (Eds.), Identification of Lichen Substances. Springer, Berlin, pp. 11-123.

Huovinen, K., Ahti, T., 1982. Biosequential patterns for the formation of depsides, depsidones and dibenzofurans in the genus Cladonia (lichen-forming ascomycetes). Ann. Bot. Fenn. 19, 225-234.

Huovinen, K., Ahti, T., 1986a. The composition and contents of aromatic lichen substances in the genus Cladina. Ann. Bot. Fenn $23,93-106$.

Huovinen, K., Ahti, T., 1986b. The composition and contents of aromatic lichen substances in Cladonia section Unciales. Ann. Bot. Fenn. 23, 173-188.

Huovinen, K., Ahti, T., 1988. The composition and contents of aromatic lichen substances in Cladonia section Perviae. Ann. Bot. Fenn. 23, 371-383.

Huovinen, K., Ahti, T., Stenroos, S., 1989a. The composition and contents of aromatic lichen substances in Cladonia section Helopodium and subsection Foliosae. Ann. Bot. Fenn. 26, 297-306.

Huovinen, K., Ahti, T., Stenroos, S., 1989b. The composition and contents of aromatic lichen substances in Cladonia, section Cocciferae. Ann. Bot. Fenn. 26, 133-148.

Huovinen, K., Ahti, T., Stenroos, S., 1990. The composition and contents of aromatic lichen substances in Cladonia section Cladonia and group Furcatae. Biblioth. Lichen. 38, 209-241.

Hyvönen, J., Ahti, T., Stenroos, S., Gowan, S.P., 1995. Genus Cladina and the section Unciales of the genus Cladonia (Cladoniaceae, lichenized Ascomycotina), a preliminary phylogenetic analysis. J. Hattori Bot. Lab. 78, 243-253.

Jahns, H.M., 1970a. Untersuchungen zur Entwicklungsgeschichte der Cladoniaceen unter besonderer Berücksichtigung des PodetienProblems. Nova Hedwigia 20, 1-177.

Jahns, H.M., 1970b. Induktion der Apothecienbildung bei Cladia aggregata (Sw.) Nyl. Ber. Deutsch. Bot. Ges, 83, 33-40.

Jahns, H.M., 1981. The genus Pilophorus. Mycotaxon 13, 289-330.

Jahns, H.M., Beltman, H.A., 1973. Variations in the ontogeny of fruiting bodies in the genus Cladonia and their taxonomic and phylogenetic significance. Lichenologist 5, 349-367.

Jahns, H.M., Klöckner, P., Jørgensen, P.M., Ott, S., 1995. Development of thallus and ascocarps in Stereocaulon tornense. Biblioth. Lichen. 58, 181-190. 
Kantvilas, G., Elix, J.A., 1999. Studies on the lichen genus Cladia Nyl. in Tasmania: the C. aggregata complex. Muelleria 12, 135162.

Kanz, B., von Brackel, W., Cezanne, R., Eichler, M., Hohmann, M.L., Teuber, D., Printzen, C., 2015. DNA barcodes for the distinction of reindeer lichens: a case study using Cladonia rangiferina and C. stygia. Herzogia 28, 445-464.

Katoh, K., Kuma, K., Toh, H., Miyata, T., 2005. MAFFT version 5: improvement in accuracy of multiple sequence alignment. Nucl. Acids Res. 33, 511-518.

Kauff, F., Lutzoni, F., 2002. Phylogeny of the Gyalectales and Ostropales (Ascomycota, Fungi): among and within order relationships based on nuclear ribosomal RNA small and large subunits. Mol. Phylogenet. Evol. 25, 138-156.

Kirk, P.M., Stalpers, J.A., Braun, U., Crous, P.W., Hansen, K., Hawksworth, D.L., Hyde, K.D., Lücking, R., Lumbsch, T.H., Rossman, A.Y., Seifert, K.A. and Stadler, M., 2013. A without prejudice list of generic names of fungi for protection under the International Code of Nomenclature for algae, fungi, and plants. IMA Fungus 4, 381-443.

Kluge, A.G., Wolf, A.J., 1993. Cladistics: what's in a word. Cladistics 9, 183-199.

Kotelko, R., Piercey-Normore, M.D., 2010. Cladonia pyxidata and C. pocillum; genetic evidence to regard them as conspecific. Mycologia 102, 534-545.

Kraichak, E., Huang, J.P., Nelsen, M., Leavitt, S.D., Lumbsch, H.T., 2018. A revised classification of orders and families in the two major subclasses of Lecanoromycetes (Ascomycota) based on a temporal approach. Bot. J. Linn. Soc. 188, 233-249.

Lamb, I.M., 1954. Studies in frutescent Lecideaceae (lichenized discomycetes). Rhodora 56, 137-153.

Lamb, I.M., Weber, W.A., Jahns, H.M., Huneck, S., 1972. Calathaspis: a new genus of the Lichen family Cladoniaceae. Occas. Papers Farlow Herb. Cryptog. Bot. 4, 1-12.

Lehtonen, S., 2011. Towards resolving the complete fern tree of life. PLoS One 6, e24851.

Lendemer, J.C., Hodkinson, B.P., 2009. The Wisdom of Fools: new molecular and morphological insights into the North American apodetiate species of Cladonia. Opusc. Philolichenum 7, 79-100.

Litterski, B., Ahti, T., 2004. World distribution of selected European Cladonia species. Symb. Bot. Upsal. 34, 205-236.

Liu, Y.J., Whelen, S., Hall, B.D., 1999. Phylogenetic relationships among ascomycetes: evidence from an RNA polymerase II subunit. Mol. Biol. Evol. 16, 1799-1808.

Lohtander, K., Myllys, L., Sundin, R., Källersjö, M., Tehler, A., 1998. The species pair concept in the lichen Dendrographa leucophaea (Arthoniales): analyses based on ITS sequences. Bryologist 101, 404-411.

Lücking, R., Hodkinson, B.P., Leavitt, S.D., 2016. The 2016 classification of lichenized fungi in the Ascomycota and Basidiomycota - Approaching one thousand genera. Bryologist $119,361-416$.

Lumbsch, H.T., Huhndorf, S.M., 2010. Myconet volume 14. 1. Outline of Ascomycota-2009. 2. Notes on ascomycete systematics. Nos. 4751-5113. Fieldiana (Life and Earth Sci.) 1, 164.

Lumbsch, H.T., Huhndorf, S.M., 2010. Myconet volume 14 outline of Ascomycota-2009 notes on Ascomycete systematics. Nos. 4751-5113. Fieldiana 1, 1-64.

Lumbsch, H.T., Rambold, G., Elix, J.A., 1995. Ramalinora (Ramalinaceae) - a new lichen genus from Australia. Austral. Syst. Bot. 8, 521-530.

Lumbsch, H.T., Parnmen, S., Rangsiruji, A., Elix, J.A., 2010a. Phenotypic disparity and adaptive radiation in the genus Cladia (Lecanorales, Ascomycota). Austral. Syst. Bot. 23, 239-247.

Lumbsch, H.T., Ahti, T., Parnmen, S., 2010b. (1926) Proposal to conserve Cladia against Heterodea (Ascomycota). Taxon 59, 643643.

Lutzoni, F., Kauff, F., Cox, C.J., McLaughlin, D., Celio, G., Dentinger, B., Padamsee, M., Hibbett, D., James, T.Y., Baloch, E., Grube, M., Reeb, V., Hofstetter, V., Schoch, C., Arnold,
A.E., Miadlikowska, J., Spatafora, J., Johnson, D., Hambleton, S., Crockett, M., Shoemaker, R., Sung, G.H., Lücking, R., Lumbsch, T., O'Donnell, K., Binder, M., Diederich, P., Ertz, D., Gueidan, C., Hansen, K., Harris, R.C., Hosaka, K., Lim, Y.W., Matheny, B., Nishida, H., Pfister, D., Rogers, J., Rossman, A., Schmitt, I., Sipman, H., Stone, J., Sugiyama, J., Yahr, R., Vilgalys, R., 2004. Assembling the fungal tree of life: Progress, classification, and evolution of subcellular traits. Am. J. Bot. 91, 1446-1480.

Mattick, F., 1938. Systembildung und Phylogenie der Gattung Cladonia. Beih. Bot. Centralbl. 58B, 215-234.

Mattick, F., 1940. Übersicht der Flechtengattung Cladonia in neuer systematischer Anordnung. Feddes Repert. 49, 140-168.

Miadlikowska, J., Kauff, K., Franker, E., Grube, M., Hafellner, J., Reeb, V., Hodkinson, B.P., Kukwa, M., Hestmark, G., Otálora, M.G., Rauhut, A., Büdel, B., Scheidegger, C., Timdal, E., Stenroos, S., Brodo, I., Perlmutter, G.B., Ertz, D., Diederich, P., Lendemer, J.C., May, P., Schoch, C.L., Arnold, A.E., Gueidan, C., Tripp, E., Yahr, R., Robertson, C., Lutzoni, F., 2006. New insights into classification and evolution of the Lecanoromycetes (Pezizomycotina, Ascomycota) from phylogenetic analyses of three ribosomal RNA and two protein-coding genes. Mycologia 98, 1088-1103.

Miadlikowska, J., Kauff, F., Högnabba, F., Oliver, J.C., Molnár, K., Fraker, E., Gaya, E., Hafellner, J., Hofstetter, V., Gueidan, C., Kukwa, K., Lücking, R., Björk, C., Sipman, H.J.M., Burgaz, A.R., Thell, A., Passo, A., Myllys, L., Goward, T., FernándezBrime, S., Hestmark, G., Lendemer, J., Lumbsch, H.T., Schmull, M., Schoch, C., Sérusiaux, E., Maddison, D.R., Arnold, A.E., Lutzoni, F., Stenroos, S., 2014. Multigene phylogenetic synthesis for the class Lecanoromycetes 1 (Ascomycota): 1307 fungi representing 1139 infrageneric taxa, 312 genera and 66 families. Mol. Phylogenet. Evol. 79, 132-168.

Miller, M.A., Pfeiffer, W., Schwartz, T., 2010. Creating the CIPRES science gateway for inference of large phylogenetic trees. In: Proceedings of the Gateway Computing Environments Workshop (GCE), 14 November 2010, New Orleans, LA, pp. 1-8.

Mort, M.E., Soltis, P.S., Soltis, D.E., Mabry, M.J., 2000. Comparison of three methods for estimating internal support on phylogenetic trees. Syst. Biol. 49, 160-171.

Moya, P., Škaloud, P., Chiva, S., García-Breijo, J., Reig-Armiñana, J., Vančurová, L., Barreno, E., 2015. Molecular phylogeny and ultrastructure of the lichen microalga Asterochloris mediterranea sp. nov. from Mediterranean and Canary Islands ecosystems. J. Syst. Evol. Microbiol. 65, 1838-1854.

Müller, J., 1896. Lichenes Colensoani a Reverensiss. Colenso in Nova Zelandia septentrionali prope Napier lecti, et nuperius missi, in Herbario Reg. Kewensi servati. J. Linn. Soc., Bot. 32, 197-208.

Myllys, L., Lohtander, K., Källersjö, M., Tehler, A., 1999. Sequence insertions and ITS data provide congruent information on Roccella canariensis and R. tuberculata (Arthoniales, Euascomycetes) phylogeny. Mol. Phylogenet. Evol. 12, 295-309.

Myllys, L., Stenroos, S., Thell, A., Ahti, T., 2003. Phylogeny of bipolar Cladonia arbuscula and Cladonia mitis (Lecanorales, Euascomycetes). Mol. Phylogenet. Evol. 27, 58-69.

Myllys, L., Högnabba, F., Lohtander, K., Thell, A., Stenroos, S., Hyvönen, J., 2005. Phylogenetic relationships of Stereocaulaceae based on simultaneous analysis of beta-tubulin, GAPDH and SSU rDNA sequences. Taxon 54, 605-618.

Nixon, K.C., 1999. Winclada ver. 1.00.08. Published by the author, Ithaca, NY. [Available from www.http//diversityoflife.org/winc lada/].

Nixon, K.C., Davis, J.I., 1991. Polymorphic taxa, missing values and cladistics analysis. Cladistics 7, 23-241.

Nylander, J.A.A., 2004. MrModeltest v2. Evolutionary Biology Centre, Uppsala University, Uppsala, Sweden.

Nylander, J.A., Wilgenbusch, J.C., Warren, D.L., Swofford, D.L., 2008. AWTY (are we there yet?): a system for graphical exploration of MCMC convergence in Bayesian phylogenetics. Bioinformatics 24, 581-583. 
Orange, A., James, P.W., White, F.J., 2001. Microchemical Methods for the Identification of Lichens. British Lichen Society, London.

Osyczka, P., Skubała, K., 2011. Chemical races of Cladonia cariosa and C. symphycarpa (lichenized Ascomycota) - a Polish case study in a worldwide context. Nova Hedwigia 93, 363-373.

Parnmen, S., Lumbsch, H.T., 2012. New combinations in the genus Cladia. Lichenologist 44, 297.

Parnmen, S., Rangsiruju, A., Mongkolsuk, P., Boonpragob, K., Elix, J.A., Lumbsch, H.T., 2010. Morphological disparity in Cladoniaceae: the foliose genus Heterodea evolved from fruticose Cladia species (Lecanorales, lichenized Ascomycota). Taxon 59, 841-849.

Parnmen, S., Rangsiruji, A., Mongkolsuk, P., Boonpragob, K., Nutakki, A., Lumbsch, H.T., 2012. Using phylogenetic and coalescent methods to understand the species diversity in the Cladia aggregata complex (Ascomycota, Lecanorales). PLoS One 7, e52245.

Parnmen, S., Leavitt, S.D., Rangsiruji, A., Lumbsch, H.T., 2013. Identification of species in the Cladia aggregata group using DNA barcoding (Ascomycota: Lecanorales). Phytotaxa 115, 114.

Peršoh, D., Beck, A., Rambold, G., 2004. The distribution of ascus types and photobiontal selection in Lecanoromycetes (Ascomycota) against the background of a revised SSU nrDNA phylogeny. Mycol. Progr. 3, 103-121.

Piercey-Normore, M.D., DePriest, P.T., 2001. Algal switching among lichen symbionts. Am. J. Bot. 88, 1490-1498.

Piercey-Normore, M.D., Ahti, T., Goward, T., 2010. Phylogenetic and haplotype analyses of four segregates within Cladonia arbuscula s.l. Botany 88, 397-408.

Pino-Bodas, R., Martin, M.P., Burgaz, A.R., 2010. Insight into the Cladonia convoluta-C. foliacea (Cladoniaceae, Ascomycota) complex and related species, revealed through morphological, biochemical and phylogenetic analyses. Syst. Biodivers. 8, 575586.

Pino-Bodas, R., Burgaz, A.R., Martín, M.P., Lumbsch, H.T., 2011. Phenotypical plasticity and homoplasy complicate species delimitation in the Cladonia gracilis group (Cladoniaceae, Ascomycota). Organisms Diversity Evol. 11, 343-355.

Pino-Bodas, R., Burgaz, A.R., Martin, M.P., Lumbsch, H.T., 2012a. Species delimitations in the Cladonia cariosa group (Cladoniaceae, Ascomycota). Lichenologist 44, 121-135.

Pino-Bodas, R., Martín, M.P., Burgaz, A.R., 2012b. Cladonia subturgida and C. iberica (Cladoniaceae) form a single, morphologically and chemically polymorphic species. Mycol. Progr. 11, 269-278.

Pino-Bodas, R., Martín, M.P., Burgaz, A.R., Lumbsch, H.T., $2013 \mathrm{a}$. Species delimitation in Cladonia (Ascomycota): a challenge to the DNA barcoding philosophy. Mol. Ecol. Resour. 13, 1058-1068.

Pino-Bodas, R., Ahti, T., Stenroos, S., Martín, M.P., Burgaz, A.R., 2013b. Multilocus approach to species recognition in the Cladonia humilis complex (Cladoniaceae, Ascomycota). Am. J. Bot. 100, 664-678.

Pino-Bodas, R., Burgaz, A.R., Martín, M.P., Ahti, T., Stenroos, S., Wedin, M., Lumbsch, H.T., 2015. The phenotypic features used for distinguishing species within the Cladonia furcata complex are highly homoplasious. Lichenologist 47, 287-303.

Pino-Bodas, R., Pérez-Vargas, I., Stenroos, S., Ahti, T., Burgaz, A.R., 2016. Sharpening the species boundaries in the Cladonia mediterranea complex (Cladoniaceae, Ascomycota). Persoonia 37, $1-12$.

Platnick, N.I., Griswold, C.E., Coddington, J.A., 1991. On missing entries in cladistic analysis. Cladistics 7, 337-343.

Reeb, V., Lutzoni, F., Roux, C., 2004. Contribution of RPB2 to multilocus phylogenetic studies of the euascomycetes (Pezizomycotina, Fungi) with special emphasis on the lichenforming Acarosporaceae and evolution of polyspory. Mol. Phylogenet. Evol. 32, 1036-1060.

Reinke, J., 1895. Abhandlungen über Flechten, III \& IV. Jahrb. Wiss. Bot. 28, 39-150.
Robertson, J., Piercey-Normore, M.D., 2007. Gene flow in symbionts of Cladonia arbuscula. Lichenologist 39, 69-82.

Ronquist, F., Teslenko, M., van der Mark, P., Ayres, D.L., Darling, A., Hohna, S., Larget, B., Liu, L., Suchard, M.A., Huelsenbeck, J.P., 2012. MrBayes 3.2: efficient Bayesian phylogenetic inference and model choice across a large model space. Syst. Biol. 61, 539542.

Ruoss, E., Ahti, T., 1989. Systematics of some reindeer lichens (Cladonia subg. Cladina) in the Southern Hemisphere. Lichenologist 21, 29-44.

Sandstede, H., 1931. Die Gattung Cladonia: Rabenhorst's Kryptogamen-Flora von Deutschland. Österreich, und der Schweiz, Ed. 2, 9(4/2). Akademische Verlagsgesellschaft, Leipzig.

Schoch, C.L., Sung, G.H., López-Giráldez, F., Townsend, J.P., Miadlikowska, J., Hofstetter, V., Robbertse, B., Matheny, P.B., Kauff, F., Wang, Z., Gueidan, C., Andrie, R.M., Trippe, K., Ciufetti, L.M., Wynns, A., Fraker, E., Hodkinson, B.P., Bonito, G., Groenewald, J.Z., Arzanlou, M., de Hoog, G.S., Crous, P.W., Hewitt, D., Pfister, D.H., Peterson, K., Gryzenhout, M., Wingfield, M.J., Aptroot, A., Suh, S.O., Blackwell, M., Hillis, D.M., Griffith, G.W., Castlebury, L.A., Rossman, A.Y., Lumbsch, H.T., Lücking, R., Büdel, B., Rauhut, A., Diederich, P., Ertz, D., Geiser, D.M., Hosaka, K., Inderbitzin, P., Kohlmeyer, J., Volkmann-Kohlmeyer, B., Mostert, L., O’Donnell, K., Sipman, H., Rogers, J.D., Shoemaker, R.A., Sugiyama, J., Summerbell, R.C., Untereiner, W., Johnston, P.R., Stenroos, S., Zuccaro, A., Dyer, P.S., Crittenden, P.D., Cole, M.S., Hansen, K., Trappe, J.M., Yahr, R., Lutzoni, F., Spatafora, J.W., 2009. The Ascomycota tree of life: a phylumwide phylogeny clarifies the origin and evolution of fundamental reproductive and ecological traits. Syst. Biol. 58, 224-239.

Simmons, M.P., Pickett, K.M., Miya, M., 2004. How meaningful are Bayesian support values?. Mol. Biol. Evol. 21, 188-199.

Smith, R.J., Arvidson, R., Bono, G., Chipman, A., Corkery, J., DiMeglio, J., Hansen, K., Isch, K., McAlpine, J., Marks-Fife, C., Mead, D., Miller, D., Nolte, N., Ottombrino, A., Prior, T., Streich, J., Theis, S., Vandruff, S., Wesseler, C., Wesseler, K., Wiseman, M., McCune, B., 2012. Rare inland reindeer lichens at Mima Mounds in southwest Washington State. North Amer. Fungi 7, 1-25.

Soltis, P.S., Soltis, D.E., 2003. Applying the bootstrap in phylogeny reconstruction. Statist. Sci. 18, 256-267.

Stamatakis, A., Hoover, P., Rougemont, J., 2008. A rapid bootstrap algorithm for the RAxML web servers. Syst. Biol. 57, 758-771.

Steinová, J., Stenroos, S., Grube, M., Škaloud, P., 2013. Genetic diversity and species delimitation of the zeorin-containing redfruited Cladonia species (lichenized Ascomycota) assessed with ITS rDNA and $\beta$-tubulin data. Lichenologist 45, 665-684.

Stenroos, S., 1986a. The family Cladoniaceae in Melanesia. 2. Cladonia section Cocciferae. Ann. Bot. Fenn. 23, 239-250.

Stenroos, S., 1986b. The family Cladoniaceae in Melanesia 1. Cladonia section Unciales. Ann. Bot. Fenn. 23, 161-164.

Stenroos, S., 1988. The family Cladoniaceae in Melanesia. 3. Cladonia sections Helopodium, Perviae and Cladonia. Ann. Bot. Fenn. 25, 117-148.

Stenroos, S., 1991. Status of four species of Cladonia endemic to the Madagascan Region. Ann. Bot. Fenn. 28, 107-110.

Stenroos, S., 1993. Taxonomy and distribution of the lichen family Cladoniaceae in the Antarctic and peri-Antarctic regions. Cryptog. Bot. 3, 310-344.

Stenroos, S., DePriest, P., 1998. SSU rDNA phylogeny of cladoniiform lichens. Am. J. Bot. 85, 1548-1559.

Stenroos, S., Ahti, T., Hyvönen, J., 1997. Phylogenetic analysis of the genera Cladonia and Cladina (Cladoniaceae, lichenized Ascomycota). Pl. Syst. Evol. 207, 43-58.

Stenroos, S., Hyvönen, J., Myllys, L., Thell, A., Ahti, T., 2002a. Phylogeny of the genus Cladonia s.l. (Cladoniaceae, Ascomycetes) inferred from molecular, morphological, and chemical data. Cladistics 18, 237-278.

Stenroos, S., Myllys, L., Thell, A., Hyvönen, J., 2002b. Phylogenetic hypotheses: Cladoniaceae, Stereocaulaceae, Baeomycetaceae, and Icmadophilaceae revisited. Mycol. Progr. 1, 267-282. 
Stenroos, S., Pino-Bodas, R., Weckman, D., Ahti, T., 2015. Phylogeny of Cladonia uncialis (Cladoniaceae, Lecanoromycetes) and its allies. Lichenologist, 47, 215-231.

Stiller, J.W., Hall B.D., 1997. The origin of red algae: Implications for plastid evolution. PNAS 94, 4520-4525.

Swofford, D.L., 2003. PAUP: Phylogenetic Analysis Using Parsimony (and Other Methods). Version 4.0b10.

Talavera, G., Castresana, J., 2007. Improvement of phylogenies after removing divergent and ambiguously aligned blocks from protein sequence alignments. Syst. Biol. 56, 564-577.

Tehler, A., 1996. Systematics, phylogeny and classification. In: Nash, T.H. (Ed.), Lichen Biology, Cambridge University Press, Cambridge, pp. 217-239.

Thomson, J.W., 1968 ['1967']. The Lichen Genus Cladonia in North America. University of Toronto Press, Toronto.

Tschermak-Woess, E., 1980. Asterochloris phycobiontica, gen. et spec. nov., der Phycobiont der Flechte Varicellaria carneonivea. Pl. Syst. Evol. 135, 279-294.

Vaidya, G., Lohman, D.J., Meier, R., 2011. SequenceMatrix: concatenation software for the fast assembly of multi-gene datasets with character set and codon information. Cladistics 27, 171-180.

Vainio, E.A., 1880. Tutkimus Cladoniain phylogenetillisestä kehityksestä. Frenckell \& Poika, Helsinki.

Vainio, E.A., 1887. Monographia Cladoniarum universalis. 1. Acta Soc. Fauna Fl. Fenn. 4, 1-509.

Vainio, E.A., 1894. Monographia Cladoniarum universalis. 2. Acta Soc. Fauna Fl. Fenn. 10, 1-498.

Vainio, E.A., 1897. Monographia Cladoniarum universalis. 3. Acta Soc. Fauna Fl. Fenn. 14, 1-268.

Verdon, D., Elix, J.A., 1986 ['1987'] Myelorrhiza, a new Australian lichen genus from North Queensland. Brunonia 9, 193-214.

Wedin, M., Döring, H., 1999. The phylogenetic relationship between the Sphaerophoraceae, Neophyllis and Austropeltum (lichenised Ascomycota). Mycol. Res. 103, 1131-1137.

Wedin, M., Döring, H., Ekman, S., 2000. Molecular phylogeny of the lichen families Cladoniaceae, Sphaerophoraceae, and Stereocaulaceae (Lecanorales, Ascomycotina). Lichenologist 32, 171-187.

Wei, J.C., Ahti, T., 2002. Cetradonia, a new genus in the new family Cetradoniaceae (Lecanorales, Ascomycota). Lichenologist 34, $19-31$.

Wheeler, W.C., 2012. Systematics: A Course of Lectures. John Wiley and Sons, Chichester.

Wheeler, W.C., Coddington, J.A., Crowley, L.M., Dimitrov, D., Goloboff, P.A., Griswold, C.E., Hormiga, G., Prendini, L., Ramírez, M.J., Sierwald, P., Almeida-Silva, L., Alvarez-Padilla, F., Arnedo, M.A., Benavides, L.R., Benjamin, S.P., Bond, J.E., Grismado, C.J., Hasanf, E., Hedin, M., Izquierdo, M.A., Labarque, F.M., Ledford, J., Lopardo, L., Maddison, W.P., Miller, J.A., Piacentini, L.N., Platnick, N.I., Polotow, D., SilvaDávila, D., Scharff, N., Szüts, T., Ubick, D., Vink, C.J., Wood, H.M., Zhang, J., 2017. The spider tree of life: phylogeny of Araneae based on target-gene analyses from an extensive taxon sampling. Cladistics 33, 574-616.

White, F.J., James, P.W., 1985. A new guide to microchemical techniques for the identification of lichen substances. British Lichen Society Bulletin 57 (Supplement), 1-41.

White, T.J., Bruns, T., Lee, S., Taylor, J., 1990. Amplification and direct sequencing of fungal ribosomal RNA genes for phylogenetics. In: Innis, M.A., Gelfand, D.H., Sninsky, J.J.,
White, T.J. (Eds.), PCR Protocols: A Guide to Methods and Applications, Academic Press, San Diego, CA, pp. 315-322.

Wijayawardene, N.N., Hyde, K.D., Rajeshkumar, K.C., Hawksworth, D.L., Madrid, H., Kirk, P.M., et al., 2017. Notes for genera: Ascomycota. Fungal Divers 86, 1-594.

Wijayawardene, N.N., Hyde, K.D., Lumbsch, H.T., Liu, J.K., Maharachchikumbura, S.S., Ekanayaka, A.H., Tian, Q., Phookamsak, R., 2018. Outline of Ascomycota: 2017. Fungal Divers. 88, 167-263.

Wiklund, E., Wedin, M., 2003. The phylogenetic relationships of the cyanobacterial lichens in the Lecanorales suborder Peltigerineae. Cladistics 19, 419-431.

Wirtz, N., Printzen, C., Lumbsch, H.T., 2008. The delimitation of Antarctic and bipolar species of neuropogonoid Usnea (Ascomycota, Lecanorales): a cohesion approach of species recognition for Usnea perpusilla complex. Mycol. Res. 112, 472484.

Wolsan, M., Sato, J.J., 2010. Effects of data incompleteness on the relative performance of parsimony and Bayesian approaches in a supermatrix phylogenetic reconstruction of Mustelidae and Procyonidae (Carnivora). Cladistics 26, 168-194.

Yahr, R., Vilgalys, R., DePriest, P.T., 2006. Geographic variation in algal partners of Cladonia subtenuis (Cladoniaceae) highlights the dynamic nature of a lichen symbiosis. New Phytol. 171, 847-860.

Zahlbrucker, A., 1926. Lichenes. In: Engler, A., Prantl, K. (Eds), Die Natürlichen Pflanzenfamilien, vol. 2, Bd. 8. Verlag von Wilhelm Engelmann, Leipzig, pp. 61-270.

Zenker, K.C.T., 1827-1829. Lichenes. In: Goebel, F. (Ed.), Pharmaceutische Waarenkunde Vol. 1, Bärecke, Eisenach, pp. $1-200$.

Zhou, Q., Wei, J., Ahti, T., Stenroos, S., Högnabba, F., 2006. The systematic position of Gymnoderma and Cetradonia based on SSU rDNA sequences. J. Hattori Bot. Lab., 100, 871-880.

\section{Supporting Information}

Additional supporting information may be found online in the Supporting Information section at the end of the article.

Fig. S1. Phylogeny of Cladoniaceae based on ITS rDNA.

Fig. S2. Phylogeny of Cladoniaceae based on IGS rDNA.

Fig. S3. Phylogeny of Cladoniaceae based on $E F-1 \alpha$.

Fig. S4. Phylogeny of Cladoniaceae based on $R P B 2$.

Fig. S5. Phylogeny of Cladoniaceae based on RPBI.

Fig. S6. Strict consensus tree of MP analysis of TNT based on the $5+4+3+2+1$ dataset (ITS rDNA, IGS rDNA, $E F-1 \alpha$, RPB1 and $R P B 2$ ).

Table S1. List of specimens used in this study with voucher information and GenBank accession numbers. New sequences are indicated in bold type. 